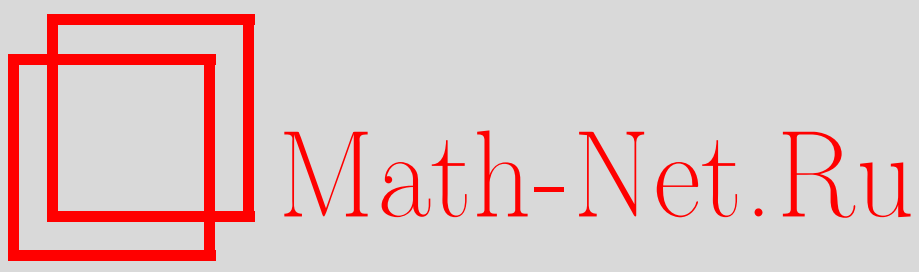

В. В. Жиков, Об одном расширении и применении метода двухмасштабной сходимости, Матем. сб., 2000, том 191, номер 7, 31-72

DOI: https://doi.org/10.4213/sm491

Использование Общероссийского математического портала Math-Net.Ru подразумевает, что вы прочитали и согласны с пользовательским соглашением

http: //www. mathnet.ru/rus/agreement

Параметры загрузки:

IP: 54.92 .164 .108

26 апреля 2023 г., 12:34:46 
УДК 517.9

\title{
В.В. Жиков \\ Об одном расширении и применении метода двухмасштабной сходимости
}

\begin{abstract}
Вводится понятие двухмасштабной сходимости, связанной с данной периодической борелевской мерой $\mu$. В том случае, когда $d \mu=d x$-мера Лебега на торе, получаем сходимость в смысле Nguetseng-Allaire. Главные свойства двухмасштабной сходимости обнаруживаются при совместном рассмотрении последовательности функций и последовательности их градиентов. Дается приложение двухмасштабной сходимости к усреднению некоторых задач из теории пористых сред (модели double-porosity). Формулируется математическое представление о "параллельных потоках, мягко или слабо связанных между собой". Строится усредненный оператор, а сам результат о сходимости интерпретируется как "силшная двухмасштабная резольвентная сходимость". В связи с этим затрагиваются вопросы поведения спектра при усреднении.

Библиография: 25 названий.
\end{abstract}

\section{§1. Введение}

1.1. Моделью усреднения служит задача Дирихле для дивергентного эллиптического уравнения с периодическими быстроосциллирующими коэффициентами:

$$
\begin{gathered}
-\operatorname{div}\left(A\left(\varepsilon^{-1} x\right) \nabla u_{\varepsilon}\right)+\lambda u_{\varepsilon}=f \quad \text { в } \Omega, \\
u_{\varepsilon} \in H_{0}^{1}(\Omega), \quad \lambda>0 .
\end{gathered}
$$

Здесь $\Omega$ - ограниченная область в $\mathbb{R}^{N}, A(y)$ - периодическая измеримая матрица, удовлетворяющая условию эллиптичности и ограниченности:

$$
\alpha I \leqslant A \leqslant \alpha^{-1} I, \quad \alpha>0 .
$$

Впервые теорема об усреднении была доказана в 1973 г. Де Джорджи и Спаньоло [1]. Доказательство было непрямым и довольно сложным. Вскоре Н. С. Бахвалов, а также Ж. Лионс и другие получили прямые доказательства, основанные на применении мошных средств асимптотического анализа, когда решение ищется в виде

$$
u(x, y)+\varepsilon u_{1}(x, y)+\cdots, \quad y=\varepsilon^{-1} x,
$$

где $u, u_{1}$ периодичны по $y$, см. [2], [3]. Асимптотический анализ позволил не только найти усредненное уравнение и доказать собственно усреднение, т.е. сходимость

Работа вьполнена при финансовой поддержке Российского фонда фундаментальных исследований (грант № 99-01-00072). 
решения $u_{\varepsilon}$ к решению усредненного уравнения, но и оценить разность между точным решением и эффективно строящимся первым приближением. Метод асимптотических разложений остается главньм орудием теории усреднения и в настоящее время. Часто его применяют только для формального нахождения усредненного уравнения и первого приближения, а для строгого доказательства сходимости используют метод компенсированной компактности Тартара-Мюра.

В 1989 г. G. Nguetseng [4] высказал интересную идею двухмасштабной сходимости, что фактически дало новый способ обоснования свойств усреднения. Метод двухмасштабной сходимости был далее развит в работах G. Allaire [5]. Этот метод оказался полезным в задачах, имеюших более сложную структуру, чем стандартная модель усреднения. В стандартной модели мы имеем дело с ограниченной в $H_{0}^{1}(\Omega)$ последовательностью решений $u_{\varepsilon}$. Поэтому естественно, что нулевое приближение $u(x, y)$ в (1.3) не зависит от $y, u(x)$ - это сильный $L^{2}$-предел последовательности $u_{\varepsilon}$. Между тем имеются многочисленные модели, в которых последовательность $u_{\varepsilon}$ ограничена, но не компактна в $L^{2}(\Omega)$. В этой ситуации слабьй $L^{2}$-предел последовательности $u_{\varepsilon}$ нельзя считать удовлетворительньм приближением к $u_{\varepsilon}$ при малых $\varepsilon$ и предпочтительней иметь дело с двухмасштабным пределом - функцией $u(x, y)$ двух переменных $x, y$, периодичной по $y$.

Поясним сказанное на простом примере. Рассмотрим задачу Дирихле

$$
u_{\varepsilon} \in H_{0}^{1}(\Omega), \quad-\varepsilon^{2} \Delta u_{\varepsilon}+u_{\varepsilon}=f(x) a\left(\varepsilon^{-1} x\right),
$$

где $f(x) \in C_{0}^{\infty}(\Omega), a(y)$ - ограниченная периодическая функция, $a \not \equiv$ const. Из интегрального тождества

$$
\varepsilon^{2} \int_{\Omega} \nabla u_{\varepsilon} \cdot \nabla \psi d x+\int_{\Omega} u_{\varepsilon} \psi d x=\int_{\Omega} f(x) a\left(\varepsilon^{-1} x\right) \psi(x) d x, \quad \psi \in C_{0}^{\infty}(\Omega),
$$

видно, что $u_{\varepsilon}, \varepsilon \nabla u_{\varepsilon}$ ограничены в $L^{2}(\Omega)$. Поэтому первое слагаемое в левой части сходится к нулю при $\varepsilon \rightarrow 0$. Предельный переход в тождестве дает уравнение

$$
\bar{u}(x)=\langle a\rangle f(x)
$$

в котором $\bar{u}(x)$ - слабый предел $u_{\varepsilon}(x),\langle a\rangle$ - среднее значение функции $a(y)$.

Можно ли считать, что получено "настоящее" предельное уравнение? Критерием служит свойство сходимости энергий. Энергия, отвечающая допредельному уравнению (1.4), равна

$$
E_{\varepsilon}=\varepsilon^{2} \int_{\Omega}\left|\nabla u_{\varepsilon}\right|^{2} d x+\int_{\Omega} u_{\varepsilon}^{2} d x=\int_{\Omega} f(x) a\left(\varepsilon^{-1} x\right) u_{\varepsilon}(x) d x .
$$

Ниже будет показано, что

$$
\lim _{\varepsilon \rightarrow 0} E_{\varepsilon}>\int_{\Omega} f(x) \bar{u}(x)\langle a\rangle d x=\langle a\rangle^{2} \int_{\Omega} f^{2}(x) d x
$$

т.е. сходимости энергий не наблюдается и слабый предел $\bar{u}(x)$ действительно не является удовлетворительным приближением к $u_{\varepsilon}$ при малых $\varepsilon$. 
Попробуем найти приближение к $u_{\varepsilon}$ в форме $u_{\varepsilon}^{0}(x)=u(x, y), y=\varepsilon^{-1} x$. Имеем:

$$
-\varepsilon^{2} \Delta u_{\varepsilon}^{0}+u_{\varepsilon}^{0}=-\Delta_{y} u-\varepsilon \operatorname{div}_{y} \nabla_{x} u-\varepsilon \operatorname{div}_{x} \nabla_{y} u-\varepsilon^{2} \Delta_{x} u+u
$$

Члены порядка $\varepsilon^{0}$ приравняем к $f(x) a(x)$ :

$$
-\Delta_{y} u(x, y)+u(x, y)=f(x) a(y)
$$

Это - периодическая задача относительно $y$, в которой $x$ играет роль параметра. Видим, что $u(x, y)=f(x) b(y)$, где $b$ - решение периодической задачи $-\Delta b+b=a$. Для разности $z_{\varepsilon}(x)=u_{\varepsilon}(x)-u\left(x, \varepsilon^{-1} x\right)$ имеем

$$
z_{\varepsilon} \in H_{0}^{1}(\Omega), \quad-\varepsilon^{2} \Delta z_{\varepsilon}+z_{\varepsilon}=2 \varepsilon \nabla f \cdot \nabla b+\varepsilon^{2} b \Delta f=g_{\varepsilon} .
$$

Так как $\left|z_{\varepsilon}\right| \leqslant c$, то

$$
\varepsilon^{2} \int_{\Omega}\left|\nabla z_{\varepsilon}\right|^{2} d x+\int_{\Omega} z_{\varepsilon}^{2} d x=\int_{\Omega} g_{\varepsilon} z_{\varepsilon} d x \leqslant c_{1} \varepsilon
$$

в частности,

$$
\lim _{\varepsilon \rightarrow 0} \int_{\Omega}\left|u_{\varepsilon}(x)-u\left(x, \varepsilon^{-1} x\right)\right|^{2} d x=0 .
$$

Не будем давать сейчас точного определения двухмасштабного предела, а только заметим, что соотношение (1.8) является достаточным (и весьма близким к необходимому) условием двухмасштабной сходимости $u_{\varepsilon}(x) \rightarrow u(x, y)$. Предельным уравнением следует считать уравнение (1.7) для $u$ как функции $2 N$ переменных. Сходимость энергий теперь следует из (1.8), так как

$$
\begin{aligned}
\lim _{\varepsilon \rightarrow 0} E_{\varepsilon} & =\lim _{\varepsilon \rightarrow 0} \int_{\Omega} f(x) a\left(\varepsilon^{-1} x\right) u\left(x, \varepsilon^{-1} x\right) d x \\
& =\int_{\Omega} \int_{\square} f(x) a(y) u(x, y) d x d y=\langle a b\rangle \int_{\Omega} f^{2} d x
\end{aligned}
$$

по свойству среднего значения. Из уравнения $-\Delta b+b=a$ имеем $\langle a\rangle=\langle b\rangle$, $\langle a b\rangle>\left\langle b^{2}\right\rangle>\langle b\rangle^{2}=\langle a\rangle^{2}$, и неравенство (1.6) доказано.

Соотношение (1.8) соответствует “сильной” двухмасштабной сходимости. Слабая двухмасштабная сходимость $u_{\varepsilon}(x) \stackrel{2}{\rightarrow} u(x, y)$ означает по определению

$$
\begin{gathered}
\lim _{\varepsilon \rightarrow 0} \int_{\Omega} u_{\varepsilon}(x) b\left(\varepsilon^{-1} x\right) \varphi(x) d x=\int_{\Omega} \int_{\square} u(x, y) b(y) \varphi(x) d y d x, \\
\varphi \in C_{0}^{\infty}(\Omega), \quad b \in C_{\mathrm{per}}^{\infty}
\end{gathered}
$$

где $C_{\text {per }}^{\infty}=C_{\text {per }}^{\infty}(\square)$ - пространство бесконечно дифференцируемых периодических функций, $\square=[0,1)^{N}-$ ячейка периодичности. 
Относительно слабой двухмасштабной сходимости доказан целый ряд свойств, позволяюших переходить к пределу в интегральных тождествах. Одно из них состоит в следуюшем: если $u_{\varepsilon}, \varepsilon \nabla u_{\varepsilon}$ ограничены в $L^{2}(\Omega)$, то (с точностью до выделения подпоследовательности)

$$
\begin{gathered}
u_{\varepsilon}(x) \stackrel{2}{\rightarrow} u(x, y) \in L^{2}\left(\Omega, H_{\mathrm{per}}^{1}\right), \\
\varepsilon \nabla u_{\varepsilon}(x) \stackrel{2}{\rightarrow} \nabla_{y} u(x, y),
\end{gathered}
$$

где $H_{\mathrm{per}}^{1}=H_{\mathrm{per}}^{1}(\square)$ - соболевское пространство периодических функций. Посмотрим, как с помощью этого свойства вывести предельное уравнение (1.7).

Возьмем в тождестве (1.5) пробную функцию

$$
\psi(x)=\psi_{\varepsilon}(x)=\varphi(x) w(y), \quad \text { где } y=\varepsilon^{-1} x, \quad \varphi \in C_{0}^{\infty}(\Omega), \quad w \in C_{\mathrm{per}}^{\infty}(\square) .
$$

Тогда

$$
\begin{aligned}
\int_{\Omega} \varepsilon \nabla u_{\varepsilon}(x) \cdot & \nabla_{y} w\left(\varepsilon^{-1} x\right) \varphi(x) d x+\varepsilon^{2} \int_{\Omega} \nabla u_{\varepsilon}(x) \cdot \nabla \varphi(x) w\left(\varepsilon^{-1} x\right) d x \\
& +\int_{\Omega} u_{\varepsilon}(x) \varphi(x) w\left(\varepsilon^{-1} x\right) d x=\int_{\Omega} f(x) a\left(\varepsilon^{-1} x\right) w\left(\varepsilon^{-1} x\right) \varphi(x) d x .
\end{aligned}
$$

Второе слагаемое слева сходится к нулю при $\varepsilon \rightarrow 0$, а пределы остальных вычислим согласно (1.9), (1.10). В результате

$$
\int_{\Omega} \int_{\square} \nabla_{y} u \cdot \nabla_{y} w \varphi d y d x+\int_{\Omega} \int_{\square} u w \varphi d y d x=\int_{\Omega} \int_{\square} f a w \varphi d y d x .
$$

Поскольку $\varphi, w$ произвольны, отсюда получаем предельное уравнение (1.7).

1.2. За последние годы наряду с активностью в области двухмасштабной сходимости наблюдалось развитие техники усреднения и в другом направлении - геометрическом. Чтобы описать геометрическую сторону дела, удобно дать общую постановку задачи усреднения в терминах периодической меры.

Пусть $\mu$-периодическая борелевская мера на $\mathbb{R}^{N}, \square=[0,1)^{N}$ - ячейка или тор периодичности, $\int_{\square} d \mu=1$. Введем меру $\mu_{\varepsilon}$ равенством

$$
\mu_{\varepsilon}(B)=\varepsilon^{N} \mu\left(\varepsilon^{-1} B\right) \text { для любого борелевского } B \subset \mathbb{R}^{N} \text {. }
$$

Мера $\mu_{\varepsilon}$ имеет период $\varepsilon$ и

$$
\int_{\varepsilon \square} d \mu_{\varepsilon}=\varepsilon^{N} \int_{\square} d \mu=\varepsilon^{N}
$$

Отсюда следует, что $\mu_{\varepsilon}$ слабо сходится к мере Лебега: $d \mu_{\varepsilon} \rightarrow d x$, т.е.

$$
\lim _{\varepsilon \rightarrow 0} \int_{\mathbb{R}^{N}} \varphi d \mu_{\varepsilon}=\int_{\mathbb{R}^{N}} \varphi d x, \quad \varphi \in C_{0}^{\infty}\left(\mathbb{R}^{N}\right) .
$$


С мерой $\mu_{\varepsilon}$ свяжем соболевское пространство $H_{0}^{1}\left(\Omega, d \mu_{\varepsilon}\right)$, которое определим следуюшим образом. Пусть $W=W_{\varepsilon}$ - замыкание множества всех пар $\{u, \nabla u: u \in$ $\left.C_{0}^{\infty}(\Omega)\right\}$ в произведении $L^{2}\left(\Omega, d \mu_{\varepsilon}\right) \times L^{2}\left(\Omega, d \mu_{\varepsilon}\right)^{N}$. Элементами $W$ служат пары $u, z$. Условимся вектор $z$ обозначать через $\nabla u$ и назьвать градиентом $u: z=\nabla u$. Совокупность первых компонент образует соболевское пространство $H_{0}^{1}\left(\Omega, d \mu_{\varepsilon}\right)$. При таком определении градиент функции определен неоднозначно, но это особых проблем не вызывает.

Теперь можно ввести модель усреднения в классической форме:

$$
u_{\varepsilon} \in H_{0}^{1}\left(\Omega, d \mu_{\varepsilon}\right), \quad-\operatorname{div}\left(A_{\varepsilon}(x) \nabla u_{\varepsilon}\right)+\lambda u_{\varepsilon}=f_{\varepsilon},
$$

где $A_{\varepsilon}(x)=A\left(\varepsilon^{-1} x\right), A(y)$ - периодическая $\mu$-измеримая матрица, удовлетворяющая условию (1.2). По определению $u_{\varepsilon} \in H_{0}^{1}\left(\Omega, d \mu_{\varepsilon}\right)$ есть решение этого уравнения, если выполняется интегральное тождество

$$
\int_{\Omega}\left[A\left(\varepsilon^{-1} x\right) \nabla u_{\varepsilon} \cdot \nabla \psi+\lambda u_{\varepsilon} \psi\right] d \mu_{\varepsilon}=\int_{\Omega} f_{\varepsilon} \psi d \mu_{\varepsilon}, \quad \psi \in C_{0}^{\infty}(\Omega)
$$

в котором $\nabla u_{\varepsilon}-$ некоторый градиент $u_{\varepsilon}$. Существование и единственность решения как пары $u_{\varepsilon}, \nabla u_{\varepsilon}$ следует из теоремы Рисса о представлении (подробнее см. $\S 3)$.

Очевидно, что модель (1.12) переходит в классическую модель (1.1), если в качестве меры $\mu$ взять меру Лебега на торе. Подходящим выбором меры $\mu$ получаются и другие известные из физической и математической литературы модели: усреднения в перфорированных областях, диффузии на периодических решетках и поверхностях, эффективной проводимости электрических цепей, усреднения на фракталах и др. Смысл усреднения в том, что решение $u_{\varepsilon}$ задачи $(1.12)$ в определенном смысле сходится к решению усредненной задачи, связанной уже с мерой Лебега, поскольку $d \mu_{\varepsilon} \rightarrow d x$. Здесь требуется пояснить, что означает сама сходимость последовательности $u_{\varepsilon} \in L^{2}\left(\Omega, d \mu_{\varepsilon}\right)$ к функции $u \in L^{2}(\Omega, d x)=L^{2}(\Omega)$.

Будем считать, что последовательность $u_{\varepsilon}$ ограничена:

$$
\varlimsup_{\varepsilon \rightarrow 0} \int_{\Omega} u_{\varepsilon}^{2} d \mu_{\varepsilon}<\infty
$$

Тогда слабая сходимость $u_{\varepsilon} \rightarrow u \in L^{2}(\Omega)$ означает по определению, что

$$
\lim _{\varepsilon \rightarrow 0} \int_{\Omega} \varphi u_{\varepsilon} d \mu_{\varepsilon}=\int_{\Omega} \varphi u d x \quad \forall \varphi \in C_{0}^{\infty}(\Omega)
$$

Сильная сходимость $u_{\varepsilon} \rightarrow u$ означает, что

$$
\lim _{\varepsilon \rightarrow 0} \int_{\Omega} u_{\varepsilon} v_{\varepsilon} d \mu_{\varepsilon}=\int_{\Omega} u v d x, \text { как только } v_{\varepsilon} \rightarrow v
$$


ПРЕДЛОЖЕНИЕ 1.1. Всякая ограниченная последовательность компактна относительно слабой сходимости. Кроме того, сильная сходимость $u_{\varepsilon} \rightarrow u$ складывается из слабой сходимости $u_{\varepsilon} \rightarrow u$ и соотношения

$$
\lim _{\varepsilon \rightarrow 0} \int_{\Omega} u_{\varepsilon}^{2} d \mu_{\varepsilon}=\int_{\Omega} u^{2} d x .
$$

Эти свойства, доказанные в [6], легко следуют из аналогичных свойств двухмасштабной сходимости, которые будут установлены в $§ 2$.

В работах [7], [8] показано, что для обоснования усреднения требуется лишь одно свойство меры $\mu-$ связность или әргодичность. По определению мера $\mu$ связна (на торе периодичности) или эргодична, если $u \equiv$ const $\mu$-п.в., как только найдутся $u_{n} \in C_{\text {per }}^{\infty}(\square)$ :

$$
\lim _{n \rightarrow \infty} \int_{\square}\left|u_{n}-u\right|^{2} d \mu=0, \quad \lim _{n \rightarrow \infty} \int_{\square}\left|\nabla u_{n}\right|^{2} d \mu=0 .
$$

Мера Лебега $d x$, очевидно, эргодична: функция $u$ из (1.15) принадлежит соболевскому пространству $H_{\text {per }}^{1}(\square)$ на торе, имеет нулевой градиент и поэтому является константой п.в. В общем случае эргодичность меры $\mu$ означает, что функция $u$ есть константа, если она принадлежит соболевскому пространству $H_{\mathrm{per}}^{1}(\square, d \mu)$ и обладает нулевым "градиентом", см. $\S 3$. Во многих случаях эргодичность меры легко устанавливается. Достаточным признаком эргодичности служит выполнение неравенства Пуанкаре

$$
\int_{\square} \varphi^{2} d \mu \leqslant C\left\{\left(\int_{\square} \varphi d \mu\right)^{2}+\int_{\square}|\nabla \varphi|^{2} d \mu\right\}, \quad \varphi \in C_{\mathrm{per}}^{\infty}(\square) .
$$

Свойство эргодичности всегда обеспечивает усреднение, но при этом усредненная матрица не обязательно положительно определена.

Рассмотрим некоторые примеры эргодических мер.

$1^{\circ}$. Вырождаюшиеся эллиптические операторы. Пусть $\mu$ абсолютно непрерывна относительно меры Лебега на торе, $d \mu=\rho d x$. Мера $\mu$ будет эргодичной, а усредненная матрица - невырожденной, если $\int_{\square} \rho^{-1} d x<\infty$, см. [7], [8].

$2^{\circ}$. Перфорированные области. Пусть $F$ - открытое периодическое множество в $\mathbb{R}^{N}$ и

$$
d \mu=\rho d x, \quad \rho(x)= \begin{cases}\frac{1}{|\square \cap F|} & \text { в } F \\ 0 & \text { вне } F .\end{cases}
$$

В данном случае $d \mu_{\varepsilon}=\rho\left(\varepsilon^{-1} x\right) d x$ и интегрирование в (1.13) ведется только по $\Omega_{\varepsilon}=\varepsilon F \cap \Omega$ - “перфорированной области”. Само решение $u_{\varepsilon}$ задачи (1.12) определено, очевидно, только в перфорированной области. Усреднению в перфорированных областях посвяшена обширная литература, см. [9]-[11]. Классический подход основан на идее продолжения решения с перфорированной области $\Omega_{\varepsilon}$ на исходную область $\Omega$ с сохранением энергетических оценок, что требовало существенных ограничений на структуру периодического множества $F$ (условие "сильной связанности" по Хруслову). Так, давний вопрос об обосновании усреднения в 
случае, когда $F$ просто связно в $\mathbb{R}^{N}$, не поддавался решению классическими методами.

Между тем обычная связность $F$ в $\mathbb{R}^{N}$ (или даже только на торе) влечет эргодичность меры $\mu$, как это непосредственно ясно из (1.15). Построены также примеры открытых, но не связных множеств $F$, для которых соответствующая мера эргодична и усредненная матрица положительно определена [7].

$3^{\circ}$. Фрактальные меры. Пусть $N=2$ и периодическая мера $\mu$ имеет структуру

$$
d \mu=\frac{d \mu_{0}\left(x_{1}\right) \times d x_{2}+d x_{1} \times d \mu_{0}\left(x_{2}\right)}{2}
$$

где $\mu_{0}$ - некоторая нормированная мера на отрезке $[0,1]$. В частности, в качестве $\mu_{0}$ можно взять естественную меру, сосредоточенную на канторовом множестве $C \subset[0,1]$. Мера $\mu$ будет сосредоточена на множестве

$$
C \times[0,1] \cup[0,1] \times C-\text { “странной канторовой сетке". }
$$

Для этих мер доказано неравенство Пуанкаре (1.16), и поэтому они эргодичны.

$4^{\circ}$. Периодические графы. Рассмотрим на плоскости периодический связный граф, например обычную квадратную сетку. Меру $\mu$ определим как сумму линейных мер звеньев, взятых с некоторыми положительными весами. Тогда мера $\mu$ будет эргодической и невырожденной.

1.3. В рассмотренной выше модели (1.12) матрица проводимости имеет специальную структуру $A_{\varepsilon}(x)=A\left(\varepsilon^{-1} x\right)$. В приложениях существуют многочисленные задачи с более сложным вхождением малого параметра. Сейчас мы рассмотрим некоторые модели усреднения, встречающиеся в теории пористых сред.

Представим себе, что пространство $\mathbb{R}^{N}$ разбито на две периодические части $F^{\varepsilon}=\varepsilon F$ и $F_{0}^{\varepsilon}=\varepsilon F_{0}$, имеющие период $\varepsilon$. Каждая из этих частей есть отдельная пористая среда (со своей внутренней структурой, обеспечивающей фильтрацию), но коэффициент проницаемости в $F^{\varepsilon}$ имеет порядок 1 , а в $F_{0}^{\varepsilon}-$ порядок $\varepsilon^{2}$. Тогда получаем модель double-porosity. Закон Дарси, описывающий фильтрацию в такой композитной системе, приводит к дивергентному параболическому уравнению

$$
\frac{\partial u}{\partial t}=\operatorname{div}\left(a_{\varepsilon}(x) \nabla u\right), \quad a_{\varepsilon}(x)= \begin{cases}1 & \text { в } F^{\varepsilon} \\ \varepsilon^{2} & \text { в } F_{0}^{\varepsilon} .\end{cases}
$$

Вместо него можно изучать соответствуюшее резольвентное эллиптическое уравнение. Часто “жесткая" фаза $F^{\varepsilon}$ является связной в $\mathbb{R}^{N}$. Если же она состоит, скажем, из двух связных компонент, то мы имеем два “параллельных потока" в жестких фазах, связь между которыми осуществляется через "мягкую” фазу $F_{0}^{\varepsilon}$.

Вот некоторые примеры.

1. Модель двух параллельных слабо связанных потоков, см. рис. 1 . Здесь $d \mu=$ $d x$ - мера Лебега, $F_{1}, F_{2}$ - две непересекаюшиеся периодические решетки в $\mathbb{R}^{3}$, $F_{0}=\mathbb{R}^{2} \backslash F_{1} \cup F_{2}$. Коэффициент проницаемости равен единице на каждой из жестких фаз $F_{1}^{\varepsilon}=\varepsilon F_{1}, F_{2}^{\varepsilon}=\varepsilon F_{2}$ и $\varepsilon^{2}$ на мягкой фазе $F_{0}^{\varepsilon}$.

2. Простейшая модель double-porosity. Здесь $d \mu=d x-$ мера Лебега, $F_{0}-$ периодическая система шаров. При $N \geqslant 3$ шары могут иметь радиус $\frac{1}{2}$. Тогда $F_{0}$ 
- это плотная кубическая упаковка шаров. Коэффициент проницаемости равен $\varepsilon^{2}$ на $F_{0}^{\varepsilon}$ и единице на дополнении к $F_{0}^{\varepsilon}$.

3. Сеточная модель double-porosity. Здесь $\mu$ - линейная мера на периодическом графе. Сам граф и его жесткая и мягкая компоненты изображены на рис. 2.

Коэффициент проницаемости, как и в предыдущем примере, равен $\varepsilon^{2}$ на $F_{0}^{\varepsilon}$ и единице на $F_{1}^{\varepsilon}$.

4. Сеточная модель двух параллельных слабо связанных потоков. Здесь $F_{1}, F_{2}$ - две “тонкие" непересекающиеся периодические решетки в $\mathbb{R}^{3}, F_{0}$ - связываюшие их звенья, см. рис. 3.

5. “Тонкостенная модель". Здесь $\mu$ - это поверхностная мера Лебега на гранях единичного куба $\square, F_{0}$ - круги на гранях; их центры находятся в центрах граней, а радиусы $<\frac{1}{2}$, см. рис. 4 .

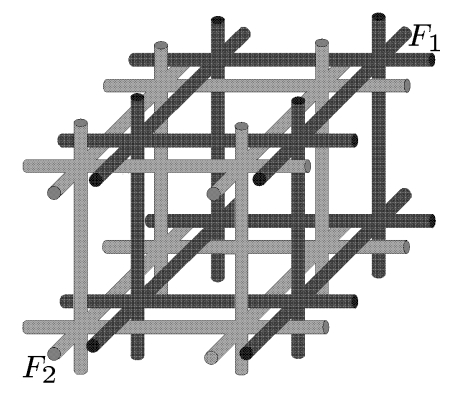

Рис. 1

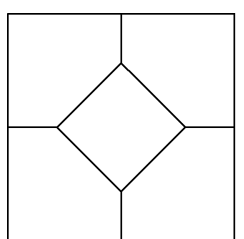

(a)

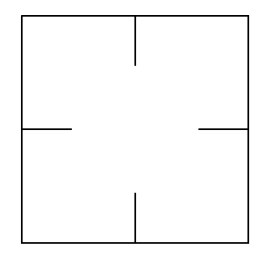

(b)

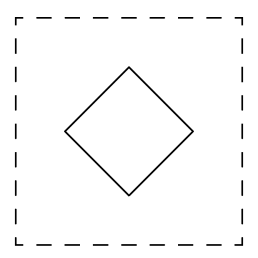

(c)

Рис. 2

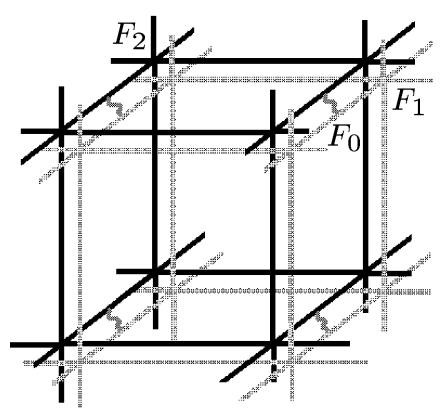

Рис. 3 


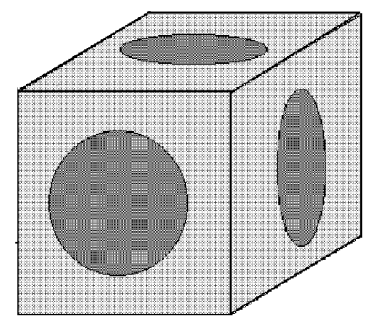

Рис. 4

Усреднению в моделях double-porosity посвящено много работ механиков и математиков [12]-[14]. Рассматривался только случай, когда $d \mu=d x$-мера Лебега, жесткая фаза $F$ связна и представляет собой достаточно гладкую периодическую область. Для нестационарного уравнения (1.17) методом Nguetseng-Allaire было выведено “предельное уравнение". Много материалов по этим вопросам имеется в книге Hornung [15]. Г.В. Сандраков [16], [17] с помощью классического метода асимптотических разложений не только доказал собственно усреднение, но и получил соответствуюшие оценки между точным решением и “двухмасштабньм" нулевым приближением. Сандракову принадлежат также интересные наблюдения в случае нескольких жестких фаз. Впрочем, применение классических методов связано с довольно сильными ограничениями гладкости на фазы.

Общая версия модели параллельных потоков в терминах меры $\mu$ дана в $\S 6$. Для исследования свойств усреднения будет применена двухмасштабная сходимость. При этом никаких ограничений гладкости на фазы не потребуется.

\section{§2. Определение двухмасштабной сходимости}

2.1. Пусть $\mu$-периодическая борелевская мера, $\mu_{\varepsilon}$ - "скейлинг"-мера, определенная равенством (1.11).

Исходнњм пунктом для двухмасштабной сходимости служит свойство среднего значения периодической функции. В простейшей форме оно выплядит так.

СвоЙСтво СРЕДНЕГО ЗНАЧенИЯ. Рассмотрим заданную на $\mathbb{R}^{N}$ периодическую $\mu$-измеримую функиию $b(x), b \in L^{1}(\square, d \mu)$. Тогда имеет место слабая сходимость мер: $b\left(\varepsilon^{-1} x\right) d \mu_{\varepsilon} \rightarrow\langle b\rangle d x$, әде $\langle b\rangle=\int_{\square} b d \mu-$ среднее значение $b$. Другими словами,

$$
\begin{aligned}
\lim _{\varepsilon \rightarrow 0} \int_{\mathbb{R}^{N}} \varphi(x) b\left(\varepsilon^{-1} x\right) d \mu_{\varepsilon} & =\int_{\mathbb{R}^{N}} \int_{\square} b(y) \varphi(x) d x d \mu(y) \\
& =\langle b\rangle \int_{\mathbb{R}^{N}} \varphi(x) d x, \quad \varphi \in C_{0}^{\infty}\left(\mathbb{R}^{N}\right) .
\end{aligned}
$$

ДокаЗАтельство. Разобьем $\mathbb{R}^{N}$ на кубы со стороной $\varepsilon$ и обозначим отдель- 
ньй такой куб символом $\varepsilon \square_{j}$. Тогда

$$
\begin{aligned}
\int_{\mathbb{R}^{N}} \varphi(x) b\left(\varepsilon^{-1} x\right) d \mu_{\varepsilon} & =\sum \int_{\varepsilon \square_{j}} \varphi(x) b\left(\varepsilon^{-1} x\right) d \mu_{\varepsilon} \\
& =\sum \varphi\left(x_{j}\right) \int_{\varepsilon \square_{j}} b\left(\varepsilon^{-1} x\right) d \mu_{\varepsilon}
\end{aligned}
$$

где $x_{j}$ - точка из куба $\varepsilon \square_{j}$. По периодичности

$$
\int_{\varepsilon \square_{j}} b\left(\varepsilon^{-1} x\right) d \mu_{\varepsilon}=\varepsilon^{N} \int_{\square} b(y) d \mu=\varepsilon^{N}\langle b\rangle .
$$

Поэтому (2.2) есть риманова сумма для интеграла (2.1), и свойство доказано.

Из (2.1) и общих свойств слабой сходимости мер получаем:

$$
\lim _{\varepsilon \rightarrow 0} \int_{\Omega} \varphi(x) b\left(\varepsilon^{-1} x\right) d \mu_{\varepsilon}=\int_{\Omega} \int_{\square} \varphi(x) b(y) d x d \mu(y),
$$

где $\Omega$ - ограниченная область, граница которой имеет нулевую лебегову меру, $\varphi$ - непрерывная функция в $\bar{\Omega}$. Ниже всегда предполагается, что $\Omega$ - липшицева область.

ЗАмЕчАНИЕ. Более общая формулировка свойства среднего значения такова. Скажем, что $\Phi=\Phi(x, y) \in C\left(\bar{\Omega}, L_{\text {per }}^{1}(\square, d \mu)\right)$, если $\Phi$ периодична по $y$ и непрерывна как функция аргумента $x \in \bar{\Omega}$ со значениями в $L^{1}(\square, d \mu)$. Тогда функция $\Phi\left(x, \varepsilon^{-1} x\right)$ будет $\mu_{\varepsilon}$-измеримой и

$$
\lim _{\varepsilon \rightarrow 0} \int_{\Omega} \Phi\left(x, \varepsilon^{-1} x\right) d \mu_{\varepsilon}=\int_{\Omega} \int_{\square} \Phi(x, y) d x d \mu .
$$

2.2. Будем изучать последовательность функций $u_{\varepsilon}(x) \in L^{2}\left(\Omega, d \mu_{\varepsilon}\right)$, всегда предполагая ее ограниченной (см. (1.14)), и использовать пробные функции

$$
\Phi(x, y)=\varphi(x) b(y), \quad \varphi \in C_{0}^{\infty}(\Omega), \quad b \in C_{\text {per }}^{\infty}(\square)
$$

ОПРЕДЕЛЕНИЕ 2.1. Последовательность $u_{\varepsilon}$ слабо двухмасштабно сходится к функции $u=u(x, y) \in L^{2}(\Omega \times \square, d x \times d \mu)=L^{2}(\Omega \times \square): u_{\varepsilon}(x) \stackrel{2}{\rightarrow} u(x, y)$, если

$$
\lim _{\varepsilon \rightarrow 0} \int_{\Omega} \Phi\left(x, \varepsilon^{-1} x\right) u_{\varepsilon}(x) d \mu_{\varepsilon}=\int_{\Omega} \int_{\square} \Phi(x, y) u(x, y) d x d \mu(y)
$$

для любой пробной функции $\Phi$ из (2.4).

Простейший пример: если $u_{\varepsilon}(x)=\Phi\left(x, \varepsilon^{-1} x\right)$, то двухмасштабньй предел равен $\Phi(x, y)$. Это следует из свойства среднего значения.

ПРЕДЛОЖЕНИЕ 2.2. Если последовательность $u_{\varepsilon}$ ограничена в $L^{2}\left(\Omega, d \mu_{\varepsilon}\right)$, то она компактна в смысле слабой двухмасштабной сходимости. 
ДокАЗАТЕльство. Множество (2.4) пробных функций сепарабельно относительно нормы $\sup _{\Omega \times \square}|\Phi|$. Выбирая счетное всюду плотное множество $Г$ пробных функций, диагональньм методом выделим подпоследовательность $\varepsilon_{k} \rightarrow 0$ такую, что предел

$$
\lim _{\varepsilon=\varepsilon_{k} \rightarrow 0} \int_{\Omega} \Phi\left(x, \varepsilon^{-1} x\right) u_{\varepsilon}(x) d \mu_{\varepsilon}=l(\Phi)
$$

существует для любой $\Phi \in \Gamma$. Но тогда этот предел, очевидно, сушествует и для любой пробной функций из (2.4). Далее, по неравенству Коши-Буняковского

$$
\begin{aligned}
\left(\int_{\Omega} \Phi\left(x, \varepsilon^{-1} x\right) u_{\varepsilon}(x) d \mu_{\varepsilon}\right)^{2} & \leqslant \int_{\Omega} u_{\varepsilon}^{2}(x) d \mu_{\varepsilon} \int_{\Omega} \Phi^{2}\left(x, \varepsilon^{-1} x\right) d \mu_{\varepsilon} \\
& \leqslant C \int_{\Omega} \Phi^{2}\left(x, \varepsilon^{-1} x\right) d \mu_{\varepsilon} .
\end{aligned}
$$

Отсюда по свойству среднего значения

$$
|l(\Phi)|^{2} \leqslant C \int_{\Omega} \int_{\square} \Phi^{2}(x, y) d x d \mu(y) .
$$

Тогда по теореме Рисса линейньй функционал $l(\Phi)$ допускает представление

$$
l(\Phi)=\int_{\Omega} \int_{\square} \Phi(x, y) u(x, y) d x d \mu(y), \quad u \in L^{2}(\Omega \times \square) .
$$

Так как линейная оболочка пробных функций плотна в $L^{2}(\Omega \times \square)$, то функция $u$ определена единственным образом. Отсюда, между прочим, ясно, что предельное соотношение (2.5) автоматически выполнено для более широкого класса пробных функций - когда $\varphi$ берется из $C(\bar{\Omega})$, a $b$ - по-прежнему из $C_{\text {per }}^{\infty}$.

Действительно, мы могли бы повторить предыдущие рассуждения для этого более широкого класса пробных функций, перейдя, быть может, в (2.6) к подпоследовательности $\varepsilon_{k}^{\prime}$. Но функция $u(x, y)$ уже определена, и поэтому в действительности переходить к подпоследовательности не нужно. Предложение доказано.

В определении 2.1 задействован минимально узкий класс пробных функций. Его можно по-разному расширять с сохранением сходимости (2.5).

ПрЕДЛОЖЕНИЕ 2.3. СХодимость (2.5) сохраняется для пробных функций $\Phi(x, y)=\varphi(x) b(y)$, әде $\varphi(x)$ берется теперь из $C(\bar{\Omega})$, а функиия $b-$ из $L^{2}(\square, d \mu)$.

ДоказАтельство. О функциях $\varphi$ уже сказано выше. Рассмотрим вопрос о расширении класса функций $b(y)$. Для $b \in L^{2}(\square, d \mu)$ возьмем

$$
\widetilde{b} \in C_{\mathrm{per}}^{\infty}(\square), \quad \int_{\square}|b-\widetilde{b}|^{2} d \mu \leqslant \delta,
$$

и воспользуемся тем, что по свойству среднего значения (2.3)

$$
\int_{\Omega}\left|b\left(\varepsilon^{-1} x\right)-\widetilde{b}\left(\varepsilon^{-1} x\right)\right|^{2} d \mu_{\varepsilon} \leqslant 2|\Omega| \delta
$$


при достаточно малом $\varepsilon$. Тогда по неравенству Коши-Буняковского

$$
\begin{gathered}
\left|\int_{\Omega} \varphi(x)\left[b\left(\varepsilon^{-1} x\right)-\widetilde{b}\left(\varepsilon^{-1} x\right)\right] u_{\varepsilon}(x) d \mu_{\varepsilon}\right| \leqslant C \delta, \\
\left|\int_{\Omega} \int_{\square} \varphi(x)[b(y)-\widetilde{b}(y)] u(x, y) d x d \mu\right| \leqslant C \delta,
\end{gathered}
$$

где $C$ не зависит от $\varepsilon$. Отсюда следует наше утверждение.

Нетрудно показать, что сходимость (2.5) сохраняется для более широкого класса "допустимых" пробных функций, например для $\Phi \in C\left(\bar{\Omega}, L_{\text {per }}^{2}(\square, d \mu)\right)$.

2.3. Перечислим некоторые свойства двухмасштабной сходимости.

i) Если $u_{\varepsilon}(x) \stackrel{2}{\rightarrow} u(x, y), a \in L_{\text {per }}^{\infty}(\square, d \mu)$, то

$$
a\left(\varepsilon^{-1} x\right) u_{\varepsilon}(x) \stackrel{2}{\rightarrow} a(y) u(x, y) .
$$

Действительно, согласно предложению 2.3 множитель $a\left(\varepsilon^{-1} x\right)$ может быть отнесен к пробной функции.

Сравним обычный слабый предел с двухмасштабным.

ii) Если $u_{\varepsilon}(x) \stackrel{2}{\rightarrow} u(x, y)$, то

$$
u_{\varepsilon}(x) \rightarrow \int_{\square} u(x, y) d \mu .
$$

В самом деле, если в (2.5) взять пробную функцию $\Phi(x, y)=\varphi(x)$, то

$$
\lim _{\varepsilon \rightarrow 0} \int_{\Omega} \varphi(x) u_{\varepsilon}(x) d \mu_{\varepsilon}=\int_{\Omega} \int_{\square} u(x, y) d \mu(y) \varphi(x) d x .
$$

Видим, что обычный слабый предел получается из двухмасштабного усреднением по периоду.

iii) Если $u_{\varepsilon}(x) \stackrel{2}{\rightarrow} u(x, y)$, то

$$
\varlimsup_{\varepsilon \rightarrow 0} \int_{\Omega} u_{\varepsilon}^{2}(x) d \mu_{\varepsilon} \geqslant \int_{\Omega} \int_{\square} u^{2}(x, y) d x d \mu .
$$

Докажем іiі). Имеем

$$
\begin{aligned}
\int_{\Omega}\left[u_{\varepsilon}(x)\right. & \left.-\Phi\left(x, \varepsilon^{-1} x\right)\right]^{2} d \mu_{\varepsilon} \\
= & \int_{\Omega} u_{\varepsilon}^{2}(x) d \mu_{\varepsilon}-2 \int_{\Omega} u_{\varepsilon}(x) \Phi\left(x, \varepsilon^{-1} x\right) d \mu_{\varepsilon}+\int_{\Omega} \Phi^{2}\left(x, \varepsilon^{-1} x\right) d \mu_{\varepsilon} .
\end{aligned}
$$

По свойству (2.5) и свойству среднего значения

$$
\varlimsup_{\varepsilon \rightarrow 0} \int_{\Omega} u_{\varepsilon}^{2} d \mu_{\varepsilon} \geqslant 2 \int_{\Omega} \int_{\square} u(x, y) \Phi(x, y) d x d \mu-\int_{\Omega} \int_{\square} \Phi^{2}(x, y) d x d \mu .
$$

Здесь $\Phi(x, y)$ - линейная комбинация пробных функций $(2.4), \Phi(x, y)=\varphi_{i}(x) b_{i}(y)$. Выбрав последовательность $\Phi_{k} \rightarrow u$ в $L^{2}(\Omega \times \square)$, получим искомое неравенство. 
ОПРЕДЕЛЕНИЕ 2.4. Последовательность $u_{\varepsilon}$ сильно двухмасштабно сходится к функции $u=u(x, y) \in L^{2}(\Omega \times \square)$, если

$\lim _{\varepsilon \rightarrow 0} \int_{\Omega} u_{\varepsilon}(x) v_{\varepsilon}(x) d \mu_{\varepsilon}=\int_{\Omega} \int_{\square} u(x, y) v(x, y) d x d \mu(y)$, как только $v_{\varepsilon}(x) \stackrel{2}{\rightarrow} v(x, y)$.

Очевидно, что сильная сходимость влечет слабую (достаточно взять $v_{\varepsilon}(x)=$ $\left.\Phi\left(x, \varepsilon^{-1} x\right)\right)$. Кроме того, взяв $v_{\varepsilon}=u_{\varepsilon}$, получим соотношение

$$
\lim _{\varepsilon \rightarrow 0} \int_{\Omega} u_{\varepsilon}^{2}(x) d \mu_{\varepsilon}=\int_{\Omega} \int_{\square} u^{2}(x, y) d x d \mu .
$$

Верно и обратное:

iv) слабая сходимость и соотношение (2.12) влекут сильную.

Чтобы убедиться в этом, рассмотрим произвольную последовательность $\varepsilon_{k} \rightarrow 0$ такую, что сушествуют пределы

$$
\lim _{\varepsilon_{k} \rightarrow 0} \int_{\Omega} u_{\varepsilon}(x) v_{\varepsilon}(x) d \mu_{\varepsilon}=\alpha, \quad \lim _{\varepsilon_{k} \rightarrow 0} \int_{\Omega} u_{\varepsilon}^{2}(x) d \mu_{\varepsilon}=\beta
$$

где $v_{\varepsilon}(x) \stackrel{2}{\rightarrow} v(x, y)$. Тогда из $(2.9),(2.12)$ получим

$$
\begin{gathered}
\lim _{\varepsilon_{k} \rightarrow 0} \int_{\Omega}\left(v_{\varepsilon}+t u_{\varepsilon}\right)^{2} d \mu_{\varepsilon} \geqslant \int_{\Omega} \int_{\square}(v+t u)^{2} d x d \mu, \\
2 t \alpha+t^{2} \beta \geqslant 2 t \int_{\Omega} \int_{\square} u v d x d \mu+t^{2} \int_{\Omega} \int_{\square} u^{2} d x d \mu .
\end{gathered}
$$

Сокрашая здесь на $t$ (сначала при $t>0$, а затем при $t<0$ ), будем иметь

$$
\alpha=\int_{\Omega} \int_{\square} u v d x d \mu
$$

что и дает (2.11).

v) Пусть $u_{\varepsilon}(x) \stackrel{2}{\rightarrow} u(x, y)$ и предельная функция $u(x, y)$ является достаточно гладкой, например $u(x, y) \in C\left(\bar{\Omega}, L_{\mathrm{per}}^{2}(\square, d \mu)\right)$. Тогда

$$
\lim _{\varepsilon \rightarrow 0} \int_{\Omega}\left|u_{\varepsilon}(x)-u\left(x, \varepsilon^{-1} x\right)\right|^{2} d \mu_{\varepsilon}=0 .
$$

Действительно, возьмем в (2.10) пробную функцию $\Phi(x, y)=u(x, y)$. Тогда

$$
\begin{aligned}
\lim _{\varepsilon \rightarrow 0} \int_{\Omega} \mid u_{\varepsilon}(x)-u & \left.\left(x, \varepsilon^{-1} x\right)\right|^{2} d \mu_{\varepsilon} \\
& =\int_{\Omega} \int_{\square} u^{2} d x d \mu-2 \int_{\Omega} \int_{\square} u u d x d \mu+\int_{\Omega} \int_{\square} u^{2} d x d \mu=0
\end{aligned}
$$

согласно (2.12), (2.5) и свойству среднего значения.

Сравним обычную сильную сходимость и двухмасштабную.

vi) Если $u_{\varepsilon}(x) \rightarrow \bar{u}(x)$, то $u_{\varepsilon}(x) \stackrel{2}{\rightarrow} \bar{u}(x)$, т.е. двухмасштабный предел не зависит от $y$. 
Докажем vi). Пусть $u_{\varepsilon}(x) \stackrel{2}{\rightarrow} u(x, y)$. Тогда по предыдущему (см. также предложение 1.1)

$$
\begin{gathered}
\bar{u}(x)=\int_{\square} u(x, y) d \mu, \\
\int_{\Omega} \bar{u}^{2}(x) d x=\lim _{\varepsilon \rightarrow 0} \int_{\Omega} u_{\varepsilon}^{2} d \mu_{\varepsilon} \geqslant \int_{\Omega} \int_{\square} u^{2}(x, y) d x d \mu \geqslant \int_{\Omega} \bar{u}^{2} d x .
\end{gathered}
$$

Ясно, что здесь наблюдаются строгие равенства и поэтому $\bar{u} \equiv u$ и $u_{\varepsilon} \stackrel{2}{\rightarrow} \bar{u}$.

Главные свойства двухмасштабной сходимости обнаруживаются при совместном рассмотрении последовательности функций $u_{\varepsilon}$ и последовательности их градиентов $\nabla u_{\varepsilon}$. Прежде чем говорить об этом, рассмотрим некоторые вопросы, связанные с гармоническим анализом на торе, наделенном мерой $\mu$.

\section{§3. Соболевские пространства}

3.1. Пусть $\mu$ - периодическая нормированная мера. Мы хотим определить соболевское пространство периодических функций $H_{\mathrm{per}}^{1}=H_{\mathrm{per}}^{1}(\square, d \mu)$.

Сначала определим пространство $W=W(\square, d \mu)$ как замькание множества пар $\left\{u, \nabla u: u \in C_{\text {per }}^{\infty}\right\}$ в $L^{2}(\square, d \mu) \times L^{2}(\square, d \mu)^{N}$.

Элементами $W$ служат пары $u, z$. Условимся вектор $z$ обозначать через $\nabla u$ и называть градиентом функции $u: z=\nabla u$. Совокупность первых компонент $u$ назовем соболевским пространством $H_{\mathrm{per}}^{1}$. Норму на этом пространстве не вводим.

Функция из соболевского пространства может иметь много градиентов.

Очевидно, что множество $Г(u)$ градиентов данной функции $u \in H_{\mathrm{per}}^{1}$ имеет структуру $\Gamma(u)=\nabla u+\Gamma(0)$, где $\nabla u-$ некоторый градиент, а $\Gamma(0)-$ множество градиентов нуля. По определению $g \in \Gamma(0)$, если

$$
\exists \varphi_{n} \in C_{\text {per }}^{\infty}: \quad \lim _{n \rightarrow \infty}\left\langle\varphi_{n}^{2}\right\rangle=0, \quad \lim _{n \rightarrow \infty}\left\langle\left|g-\nabla \varphi_{n}\right|^{2}\right\rangle=0 .
$$

Ясно, что Г(0) есть подпространство векторного пространства $\left(L^{2}\right)^{N}$.

Для иллюстрации неединственности градиента рассмотрим простейший примep.

ПримеР. Пусть $N=2, \square=[0,1] \times\left[-\frac{1}{2}, \frac{1}{2}\right)-$ ячейка периодичности, $\mu-$ линейная мера на отрезке $I=[0,1] \times\{0\}$. По определению для $u \in H_{\mathrm{per}}^{1}(\square, d \mu)$

$$
\begin{gathered}
\exists u_{n} \in C_{\mathrm{per}}^{\infty}(\square): \quad \int_{I}\left|u-u_{n}\right|^{2} d x_{1} \rightarrow 0, \\
\int_{I}\left|\frac{\partial u}{\partial x_{1}}-z_{1}\right|^{2} d x_{1} \rightarrow 0, \quad \int_{I}\left|\frac{\partial u}{\partial x_{2}}-z_{2}\right|^{2} d x_{1} \rightarrow 0 .
\end{gathered}
$$

Тогда $u$ принадлежит одномерному соболевскому пространству $H_{\mathrm{per}}^{1}(I)$, причем $z_{1}=\frac{\partial u}{\partial x_{1}}$, т.е. $\nabla u=\left\{\frac{\partial u}{\partial x_{1}}, z_{2}\right\}$. Убедимся, что компонента $z_{2}$ может быть произвольным элементом из $L^{2}(I)$, другими словами,

$$
\Gamma(0)=\{(0, \alpha)\}, \quad \alpha \in L^{2}(I)=L^{2}(\square, d \mu) .
$$


Поскольку $\Gamma(0)$ замкнуто в $L^{2}(\square, d \mu)$, то достаточно проверить, что $(0, \alpha) \in \Gamma(0)$ для $\alpha \in C_{\text {per }}^{\infty}(I)$. В этом случае в качестве $\varphi_{n}$ из $(3.1)$ можно взять $\varphi_{n}(x)=$ $\chi\left(x_{2}\right) \alpha\left(x_{1}\right)$, где $\chi\left(x_{2}\right)$ - гладкая периодическая функция, $\chi(0)=0, \chi^{\prime}(0)=1$.

Дальнейшие свойства градиента имеет смысл обсудить в связи с эллиптическими уравнениями. Пусть $A(x)$ - периодическая $\mu$-измеримая симметрическая матрица, подчиненная условию эллиптичности и ограниченности (1.2).

Скажем, что $u \in H_{\text {per }}^{1}$ есть решение периодической задачи

$$
-\operatorname{div}(A \nabla u)+\lambda u=f, \quad f \in L^{2}(\square, d \mu), \quad \lambda>0,
$$

если вьполнено интегральное тождество

$$
\langle A \nabla u \cdot \nabla \varphi\rangle+\lambda\langle u \varphi\rangle=\langle f \varphi\rangle, \quad \varphi \in C_{\mathrm{per}}^{\infty}
$$

в котором $\nabla u$ - некоторый градиент $u$. Сушествование и единственность решения как пары $u, \nabla u$ из $W$ получить совсем просто. Действительно, с помощью левой части (3.3) на пространстве $W$ задается новое скалярное произведение, причем соответствующая норма эквивалентна исходной. Поэтому достаточно сослаться на теорему Рисса о представлении линейного функционала в гильбертовом пространстве. Таким образом, единственность здесь двоякая: одна функция из $H_{\mathrm{per}}^{1}$ и лишь один ее градиент удовлетворяют тождеству (3.3).

Интересно, что градиент, участвующий в этом тождестве, с самим уравнением не связан, а определяется только матрицей $A(x)$. Действительно, в тождестве (3.3) возьмем $\varphi=\varphi_{n}-$ последовательность из (3.1). Тогда получим

$$
A \nabla u \perp \Gamma(0)
$$

и воспользуемся следующим утверждением.

ПРЕДЛОЖЕНИЕ 3.1. Всякая функиия из соболевского пространства имеет единственный градиент, удовлетворяющий условию (3.4).

ДокАЗАТЕльство. Пусть $\nabla u$-какой-то градиент $u$. В силу эллиптичности $A$ задача

$$
c \in \Gamma(0), \quad\langle A(c+\nabla u) \cdot g\rangle=0 \quad \forall g \in \Gamma(0)
$$

имеет единственное решение. Тогда $c+\nabla u$ будет искомым градиентом.

В частности, взяв в качестве $A$ единичную матрицу, найдем, что всякая функция из соболевского пространства имеет единственный градиент, удовлетворяющий условию $\nabla u \perp \Gamma(0)$. Такой градиент называется тангенциальным. Более подробно об этом см. $\S 9$.

Уравнение (3.2) легко интерпретируется с операторной точки зрения. Покажем, что оно имеет вид

$$
\mathscr{A} u+\lambda u=f
$$

где $\mathscr{A}$ - самосопряженный неотрицательный оператор в $L^{2}=L^{2}(\square, d \mu)$. С этой целью обозначим через $D(\mathscr{A})$ множество всех решений уравнения $(3.2)$, в котором правая часть $f$ пробегает $L^{2}$. Очевидное замечание: $D(\mathscr{A})$ не зависит от выбора 
$\lambda>0$. Далее, рассмотрим второй экземпляр уравнения (3.2), в котором $f$ заменено на $g$, а $u$ заменено на $v$. Поскольку в соответствующих тождествах можно брать пробные функции $\varphi \in H_{\mathrm{per}}^{1}$, то, выбрав в $(3.3) \varphi=v$, а в тождестве для $v$-пробную функцию $\varphi=u$, получим

$$
\langle f v\rangle=\langle g u\rangle .
$$

Отсюда следует, что $D(\mathscr{A})$ плотно в $L^{2}$. Действительно, взяв $g \perp D(\mathscr{A})$, найдем, что $v=0$. Интегральное тождество для $v$ принимает вид $\langle A \nabla v \cdot \nabla \varphi\rangle=\langle g \varphi\rangle$ $\forall \varphi \in H_{\text {per }}^{1}$. Полагая в нем $\varphi=v$, получаем $\nabla v=0$ и поэтому $g=0$. Плотность множества $D(\mathscr{A})$ доказана. На множестве $D(\mathscr{A})$ зададим оператор $\mathscr{A}$ равенством $\mathscr{A} u=f-\lambda u$. Тогда $\mathscr{A}+\lambda I$ обратим, обратньй - ограничен (его норма $\left.\leqslant \lambda^{-1}\right)$ и симметричен, как это следует из (3.6). Следовательно, $\mathscr{A}$ самосопряжен.

Из самого построения ясно, что разрешимость эллиптической задачи (3.2) эквивалентна разрешимости операторного уравнения (3.5).

Мы видим, что неединственность градиента не является препятствием к рассмотрению эллиптических уравнений и привносит сюда лишш небольшие нюансы. Положение упрощается еще и тем, что само условие (3.4) оказывается поточечным. Этот результат доказан в приложении к настоящей работе, см. $\S 9$.

Все сказанное вьше о пространстве $H_{\text {per }}^{1}$ и периодической задаче (3.2) справедливо для пространства $H_{0}^{1}\left(\Omega, d \mu_{\varepsilon}\right)$ и задачи Дирихле (1.12).

3.2. Определим потенциальные и соленоидальные векторы на торе периодичности. Введем пространство $V_{\text {pot }}$ потенциальных векторов как

$$
\text { замыкание множества }\left\{\nabla \varphi: \varphi \in C_{\mathrm{per}}^{\infty}(\square)\right\} \text { в } L_{\mathrm{per}}^{2}(\square, d \mu)^{N} .
$$

Потенциальный вектор не обязан иметь нулевое среднее, $\langle v\rangle \neq 0$. Более того, нетрудно показать, что если каждый потенциальный вектор имеет нулевое среднее, то $\mu$ - это мера Лебега на торе. Вектор $b \in L^{2}(\square, d \mu)^{N}$ называется соленоидальнылм, если он ортогонален всем потенциальным, т.е.

$$
\langle b \cdot \nabla \varphi\rangle=0 \quad \forall \varphi \in C_{\text {per }}^{\infty}
$$

Таким образом, имеем ортогональное разложение

$$
L^{2}(\square, d \mu)^{N}=V_{\text {pot }} \oplus V_{\text {sol }}
$$

Отметим, что в пространстве $V_{\text {pot }}$ гладкие векторы плотны по определению, но этого нельзя утверждать относительно $V_{\text {sol }}$.

Имеется естественная связь между функциями из соболевского пространства $H_{\mathrm{per}}^{1}$ и потенциальными векторами. Например, градиент любой функции из $H_{\mathrm{per}}^{1}$ есть потенциальный вектор. В общем случае потенциальный вектор может не быть градиентом функции из $H_{\mathrm{per}}^{1}$. Однако если справедливо неравенство Пуан-

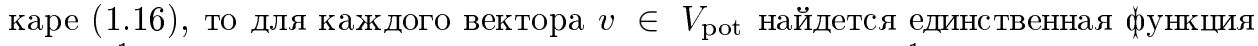

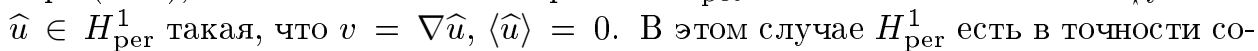
вокупность потенциалов, отвечающих векторам из $V_{\text {pot }}$.

Из ортогонального разложения (3.9) получим

$$
L^{2}(\Omega \times \square)^{N}=L^{2}\left(\Omega, L^{2}(\square)^{N}\right)=L^{2}\left(\Omega, V_{\text {pot }}\right) \oplus L^{2}\left(\Omega, V_{\text {sol }}\right)
$$


Формально $L^{2}\left(\Omega, V_{\text {pot }}\right)$ - это совокупность квадратично интегрируемых (по Бохнеру) функций аргумента $x \in \Omega$ со значениями в $V_{\text {pot }}$. Однако проще $L^{2}\left(\Omega, V_{\text {pot }}\right)$ определить как замыкание в $L^{2}(\Omega \times \square)$ линейной оболочки векторов $f(x) \nabla_{y} \varphi(y)$, где $f \in C_{0}^{\infty}(\Omega), \varphi \in C_{\mathrm{per}}^{\infty}$. Аналогично, $L^{2}\left(\Omega, V_{\mathrm{sol}}\right)$ - это замькание в $L^{2}(\Omega \times \square)$ линейной оболочки векторов $f(x) b(y)$, где $f \in C_{0}^{\infty}(\Omega), b \in V_{\text {sol }}$.

3.3. Может случиться, что ненулевой постоянный вектор является потенциальным. Обозначим через $L$ подпространство в $\mathbb{R}^{N}$, состоящее из потенциальных векторов. Мера $\mu$ называется невыро сденной, если $L=\{0\}$. Невырожденность меры тесно связана с невырожденностью усредненной матрицы, которая определяется следующим образом:

$$
A^{\mathrm{hom}} \xi \cdot \xi=\min _{v \in V_{\mathrm{pot}}}\langle A(\xi+v) \cdot(\xi+v)\rangle
$$

где, как всегда, $A=A(x)$ - периодическая $\mu$-измеримая матрица, удовлетворяющая условию (1.2). Отсюда ясно, что $A^{\text {hom }}$ (как неотрицательный самосопряженньй оператор в $\mathbb{R}^{N}$ ) имеет своим ядром подпространство $L$, а областью значений - ортогональное дополнение $\widetilde{L}=L^{\perp}$. Уравнение Эйлера для (3.11) имеет вид

$$
v \in V_{\mathrm{pot}}, \quad\langle A(\xi+v) \cdot h\rangle=0 \quad \forall h \in V .
$$

Из него

$$
\begin{gathered}
A^{\text {hom }} \xi \cdot \xi=\langle A(\xi+v) \cdot(\xi+v)\rangle=\langle A(\xi+v)\rangle \cdot \xi, \\
A^{\text {hom }} \xi=\langle A(\xi+v)\rangle, \quad A(\xi+v) \in V_{\text {sol }} .
\end{gathered}
$$

Каждый вектор из $\widetilde{L}$ является средним значением некоторого соленоидального вектора - это ясно из (3.13). Верно и обратное: среднее значение любого соленоидального вектора принадлежит $\widetilde{L}$. Действительно, если $b \in V_{\mathrm{sol}}$, то $0=\langle b \cdot \xi\rangle=$ $\langle b\rangle \cdot \xi$ для $\xi \in L$. Итак, справедливо

ПРЕДЛОЖЕНИЕ 3.2. Пусть $L$ - подпространство в $\mathbb{R}^{N}$, состоящее из потенциальных векторов, $\widetilde{L}$ - его ортогональное дополнение. Тогда усредненная матрица является нулевой на $L$ и положительно определенной на $\widetilde{L}$. Пространство $\widetilde{L}$ - это в точности совокупность средних значений соленоидальных векторов.

Сама по себе невырожденность меры $\mu$ не означает ничего глубокого с точки зрения усреднения. Для усреднения требуется другое свойство - эргодичность.

ОПРЕДЕЛЕНИЕ 3.3. Мера $\mu$ эргодична, если любая функция $u \in H_{\mathrm{per}}^{1}$, обладающая нулевым градиентом, есть константа $\mu$-почти всюду.

Сейчас мы приведем одно важное следствие эргодичности.

Для $a \in L^{2}(\square, d \mu), b \in L^{2}(\square, d \mu)^{N}$ мы пишем $a=\operatorname{div} b$, если

$$
\langle b \cdot \nabla \varphi\rangle=-\langle a \varphi\rangle, \quad \varphi \in C_{\text {per }}^{\infty}
$$

Очевидно, что функция $а$ обязана иметь нулевое среднее: $\langle a\rangle=0$. 
ЛЕММА ОБ АППРОКСИМАЦИИ [7], [8]. Если мера $\mu$ әргодична, то множсество функиий $a \in L^{2}(\square, d \mu)$, допускающих представление $a=\operatorname{div} b, b \in L^{2}(\square, d \mu)^{N}$, плотно в подпространстве всех функиий из $L^{2}(\square, d \mu)$, имеющих нулевое среднее.

В случае выполнения неравенства Пуанкаре (1.16) всякий вектор $a \in L^{2}$ с нулевьм средним допускает указанное представление $a=\operatorname{div} b$.

\section{§4. Эргодичность и двухмасштабная сходимость}

4.1. Изучим последовательность $u_{\varepsilon} \in C_{0}^{\infty}(\Omega)$, считая, что она ограничена в $L^{2}\left(\Omega, d \mu_{\varepsilon}\right)$ и $u_{\varepsilon}(x) \stackrel{2}{\rightarrow} u(x, y)$. Первый вопрос: когда двухмасштабный предел не зависит от $y$ ? Ответ сушественно использует эргодичность меры $\mu$.

ТЕОРемА 4.1. Пусть мера $\mu$ әргодична и выполнены условия

i) $u_{\varepsilon}(x) \stackrel{2}{\rightarrow} u(x, y)$;

ii) $\varepsilon\left\|\nabla u_{\varepsilon}\right\|_{L^{2}\left(\Omega, d \mu_{\varepsilon}\right)} \rightarrow 0$.

Тогда двухмасштабный предел $и(x, y)$ не зависит от $y: u(x, y)=u(x)$.

ДоказАтельство. Возьмем $a=\operatorname{div} b, a \in L^{2}(\square, d \mu), b \in L^{2}(\square, d \mu)^{N}$.

Сначала заметим, что тождество (3.14) на периодических функциях влечет за собой аналогичное тождество на финитных функциях:

$$
\varepsilon \int_{\mathbb{R}^{N}} b\left(\varepsilon^{-1} x\right) \cdot \nabla \psi(x) d \mu_{\varepsilon}=-\int_{\mathbb{R}^{N}} a\left(\varepsilon^{-1} x\right) \psi(x) d \mu_{\varepsilon}, \quad \psi \in C_{0}^{\infty}\left(\mathbb{R}^{N}\right) .
$$

Поэтому для $\varphi \in C_{0}^{\infty}(\Omega)$

$$
\begin{aligned}
& \varepsilon \int_{\Omega} \nabla u_{\varepsilon}(x) \varphi(x) \cdot b\left(\varepsilon^{-1} x\right) d \mu_{\varepsilon} \\
& \quad=\varepsilon \int_{\Omega} \nabla\left(\varphi u_{\varepsilon}\right) \cdot b\left(\varepsilon^{-1} x\right) d \mu_{\varepsilon}-\varepsilon \int_{\Omega} u_{\varepsilon}(x) \nabla \varphi(x) \cdot b\left(\varepsilon^{-1} x\right) d \mu_{\varepsilon} \\
& =-\int_{\Omega} \varphi(x) a\left(\varepsilon^{-1} x\right) u_{\varepsilon}(x) d \mu_{\varepsilon}-\varepsilon \int_{\Omega} u_{\varepsilon}(x) \nabla \varphi(x) \cdot b\left(\varepsilon^{-1} x\right) d \mu_{\varepsilon}
\end{aligned}
$$

Левая часть стремится к нулю в силу условия іi). Второе слагаемое справа также имеет нулевой предел. Поэтому

$$
0=\int_{\Omega} \int_{\square} \varphi(x) a(y) u(x, y) d x d \mu(y) .
$$

Теперь из леммы об аппроксимации следует, что $u(x, y)=u(x)$. Теорема доказана.

Пусть теперь мера $\mu$ эргодична, $u_{\varepsilon} \in C_{0}^{\infty}(\Omega), u_{\varepsilon}, \nabla u_{\varepsilon}$ ограничены в $L^{2}\left(\Omega, d \mu_{\varepsilon}\right)$,

$$
u_{\varepsilon}(x) \stackrel{2}{\rightarrow} u(x), \quad \nabla u_{\varepsilon} \stackrel{2}{\rightarrow} p(x, y)
$$

Возникают вопросы: принадлежит ли $u(x)$ соболевскому пространству $H^{1}(\Omega)$ ? Как связаны $\nabla u$ и $p(x, y)$ ? 
ТЕОРема 4.2. Пусть мера $\mu$ әргодична и невырожсдена. Тогда

$$
\begin{gathered}
u_{\varepsilon}(x) \stackrel{2}{\rightarrow} u(x) \in H_{0}^{1}(\Omega) \\
\nabla u_{\varepsilon}(x) \stackrel{2}{\rightarrow} \nabla u(x)+v(x, y), \quad \text { zдe } v \in L^{2}\left(\Omega, V_{\text {pot }}\right) .
\end{gathered}
$$

ДокАЗАТЕльСтво. Для $b \in V_{\mathrm{sol}},\langle b\rangle=\eta, \varphi \in C^{\infty}(\bar{\Omega})$

$$
\begin{aligned}
\int_{\Omega} & \varphi(x) \nabla u_{\varepsilon}(x) \cdot b\left(\varepsilon^{-1} x\right) d \mu_{\varepsilon} \\
& =\int_{\Omega} \nabla\left(\varphi u_{\varepsilon}\right) \cdot b\left(\varepsilon^{-1} x\right) d \mu_{\varepsilon}-\int_{\Omega} u_{\varepsilon}(x) \nabla \varphi(x) \cdot b\left(\varepsilon^{-1} x\right) d \mu_{\varepsilon} \\
& =-\int_{\Omega} u_{\varepsilon}(x) \nabla \varphi(x) \cdot b\left(\varepsilon^{-1} x\right) d \mu_{\varepsilon}
\end{aligned}
$$

и по определению двухмасштабной сходимости

$$
\int_{\Omega} \int_{\square} p(x, y) \varphi(x) \cdot b(y) d x d \mu(y)=-\int_{\Omega} \int_{\square} u(x) \nabla \varphi(x) \cdot b(y) d x d \mu(y) .
$$

Отсюда

$$
\begin{gathered}
\int_{\Omega} u(x) \eta \cdot \nabla \varphi(x) d x=-\int_{\Omega} z(x) \varphi(x) d x, \quad \varphi \in C^{\infty}(\bar{\Omega}), \\
z(x)=\int_{\square} p(x, y) b(y) d \mu, \quad z \in L^{2}(\Omega) .
\end{gathered}
$$

Получили, что $\eta \cdot \nabla u \in L^{2}(\Omega)$ в смысле распределений, $\eta \cdot \nabla u=z(x)$.

Теперь используем невырожденность меры $\mu$. В этом случае для любого $\eta \in \mathbb{R}^{N}$ найдется $b \in V_{\text {pot }},\langle b\rangle=\eta$. Тогда $\nabla u \in L^{2}$ в смысле распределений, т.е. $u \in H^{1}(\Omega)$, и равенство (4.2) перепишется так:

$$
\begin{gathered}
\int_{\square} \int_{\Omega} p(x, y) \cdot \varphi(x) b(y) d x d \mu=\int_{\square} \int_{\Omega} \nabla u(x) \cdot b(y) \varphi(x) d x d \mu \\
\int_{\square} \int_{\Omega}[p(x, y)-\nabla u(x)] \cdot \varphi(x) b(y) d x d \mu=0 .
\end{gathered}
$$

Поскольку линейная оболочка вектор-функций $\varphi(x) b(y)$ плотна в $L^{2}\left(\Omega, V_{\mathrm{sol}}\right)$, то в силу ортогонального разложения $(3.10) p-\nabla u \in L^{2}\left(\Omega, V_{\text {pot }}\right)$.

Остается проверить, что $u \in H_{0}^{1}(\Omega)$. Из (4.3) следует, что

$$
\int_{\Omega} u \nabla \varphi d x=-\int_{\Omega} \nabla u \varphi d x \quad \forall \varphi \in C^{\infty}(\bar{\Omega})
$$

Это означает, что функция $u$, продолженная нулем на $\mathbb{R}^{N} \backslash \Omega$, принадлежит пространству $H^{1}\left(\mathbb{R}^{N}\right)$. Но тогда благодаря липшицевости $\Omega u \in H_{0}^{1}(\Omega)$ - замьканию $C_{0}^{\infty}(\Omega)$ по соболевской норме. Теорема доказана.

Рассмотрим теперь случай вырожденной меры (см. предложение 3.2). 
По предыдушему $\eta \cdot \nabla u \in L^{2}(\Omega)$ для $\eta \in \widetilde{L}$, в частности $A^{\text {hom }} \nabla u \in L^{2}(\Omega)$ и $\widetilde{\nabla} u \in L^{2}(\Omega)$, где через $\widetilde{\nabla} u$ обозначена ортогональная проекция $\nabla u$ на $\widetilde{L}$.

Невырожденной мере соответствуют соболевские пространства $H^{1}(\Omega)$ и $H_{0}^{1}(\Omega)$, а вырожденной - пространство

$$
\widetilde{H}^{1}(\Omega)=\left\{u \in L^{2}(\Omega), \widetilde{\nabla} u \in L^{2}(\Omega)^{N}\right\}
$$

с нормой $\|u\|_{L^{2}}+\|\widetilde{\nabla} u\|_{\left(L^{2}\right)^{N}}$ и его подпространство $\widetilde{H}_{0}^{1}(\Omega)=\left\{u \in \widetilde{H}^{1}(\Omega),\left.u\right|_{\partial \Omega}=0\right\}$. При этом $\left.u\right|_{\partial \Omega}=0$ означает по определению тождество (4.4), в котором $\nabla u, \nabla \varphi$ заменены на $\widetilde{\nabla} u, \widetilde{\nabla} \varphi$. Важно, что множество $C_{0}^{\infty}(\Omega)$ плотно в $\widetilde{H}_{0}^{1}(\Omega)$. Для звездной $\Omega$ этот факт доказывается обычным сглаживанием, а для липшицевой - требуются более сложные рассуждения, см. [18; с. 311].

Теперь, если действовать как при доказательстве теоремы 4.2, получим:

$$
\begin{gathered}
u_{\varepsilon}(x) \stackrel{2}{\rightarrow} u(x) \in \widetilde{H}_{0}^{1}(\Omega), \\
\nabla u_{\varepsilon}(x) \stackrel{2}{\rightarrow} \widetilde{\nabla} u(x)+v(x, y), \quad \text { где } v \in L^{2}\left(\Omega, V_{\text {pot }}\right) .
\end{gathered}
$$

До сих пор мы для простоты рассматривали последовательность $u_{\varepsilon} \in C_{0}^{\infty}(\Omega)$. Все результаты сохраняются, если брать $u_{\varepsilon} \in H_{0}^{1}\left(\Omega, d \mu_{\varepsilon}\right)$. Единственный пункт, требующий внимания, это условие ограниченности градиентов $\nabla u_{\varepsilon}$. В случае $u_{\varepsilon} \in H_{0}^{1}\left(\Omega, d \mu_{\varepsilon}\right)$ надо говорить "найдутся такие $\nabla u_{\varepsilon} \in \Gamma\left(u_{\varepsilon}\right)$, что последовательность $\nabla u_{\varepsilon}$ ограничена в $L^{2}\left(\Omega, d \mu_{\varepsilon}\right)$ ".

4.2. Выведем из полученных результатов одно следствие, которое будет рабочим инструментом в вопросах усреднения. Будем считать, что имеют место соотношения (4.1) или более общие соотношения (4.5).

ЛЕмма 4.3. Пусть дополнительно

$\lim _{\varepsilon \rightarrow 0} \int_{\Omega} A\left(\varepsilon^{-1} x\right) \nabla u_{\varepsilon}(x) \cdot \nabla_{y} w\left(\varepsilon^{-1} x\right) \varphi(x) d \mu_{\varepsilon}=0 \quad \forall \varphi \in C_{0}^{\infty}(\Omega), w \in C_{\text {per }}^{\infty}$.

Тогда имеет место слабая сходимость "потоков":

$$
A\left(\varepsilon^{-1} x\right) \nabla u_{\varepsilon}(x) \rightarrow A^{\text {hom }} \nabla u(x) .
$$

ДоКАЗАТЕЛЬСТво. Из свойств (2.7), (2.8) Двухмасштабной сходимости имеем

$$
\begin{aligned}
& A\left(\varepsilon^{-1} x\right) \nabla u_{\varepsilon}(x) \stackrel{2}{\rightarrow} A(y)[\widetilde{\nabla} u(x)+v(x, y)], \\
& A\left(\varepsilon^{-1} x\right) \nabla u_{\varepsilon}(x) \rightarrow \int_{\square} A(y)[\widetilde{\nabla} u(x)+v(x, y)] d \mu(y) .
\end{aligned}
$$

В частности, предел (4.6) равен

$$
\int_{\Omega} \int_{\square} A(y)[\widetilde{\nabla} u(x)+v(x, y)] \varphi(x) \cdot \nabla_{y} w(y) d x d \mu=0 .
$$

Поскольку $\varphi \in C_{0}^{\infty}(\Omega)$ произвольно, то

$$
\int_{\square} A(y)[\widetilde{\nabla} u(x)+v(x, y)] \cdot \nabla_{y} w(y) d \mu=0 \quad \forall w \in C_{\mathrm{per}}^{\infty} .
$$

Видим, что $v(x, y)$ есть решение периодической задачи $(3.12)$ для $\xi=\widetilde{\nabla} u(x)$, и тогда формула (3.13) дает

$$
\int_{\square} A(y)[\widetilde{\nabla} u(x)+v(x, y)] d \mu=A^{\text {hom }} \widetilde{\nabla} u(x)=A^{\text {hom }} \nabla u(x) .
$$

Теперь (4.8) означает искомую сходимость потоков. 
4.3. Установленных вьше свойств двухмасштабной сходимости достаточно, чтобы легко вывести теорему усреднения в классической постановке. Покажем, что в тождестве (1.13) можно перейти к пределу и получить усредненную задачу, связанную уже с мерой Лебега. Усредненное уравнение имеет обычный вид

$$
u \in H_{0}^{1}(\Omega), \quad-\operatorname{div}\left(A^{\mathrm{hom}} \nabla u\right)+\lambda u=f
$$

в случае невырожденной $A^{\text {hom }}$. В случае вырождения берем $\widetilde{H}_{0}^{1}(\Omega)$ вместо $H_{0}^{1}(\Omega)$.

TЕОРема 4.4. Пусть мера $\mu$ әргодична и правые части $f_{\varepsilon}$ в (1.12) слабо (сильно) сходятся $\kappa f \in L^{2}(\Omega)$. Тогда последовательность решений слабо (сильно) сходится к решению усредненного уравнения.

ДокаЗАТЕЛЬСтво. Из тождества (1.13) и ограниченности $f_{\varepsilon}$ следует, что $u_{\varepsilon}$, $\nabla u_{\varepsilon}$ ограничены в $L^{2}\left(\Omega, d \mu_{\varepsilon}\right)$. Без потери общности считаем, что имеют место соотношения (4.1) или (4.5).

Взяв в интегральном тождестве (1.13) пробную функцию

$$
\psi(x)=\varepsilon \varphi(x) w(y), \quad \text { где } y=\varepsilon^{-1} x, \quad \varphi \in C_{0}^{\infty}(\Omega), \quad w \in C_{\mathrm{per}}^{\infty},
$$

получим

$$
\begin{aligned}
\int_{\Omega} A\left(\varepsilon^{-1} x\right) \nabla u_{\varepsilon} \cdot \nabla_{y} w \varphi d \mu_{\varepsilon}+\varepsilon \int_{\Omega} A\left(\varepsilon^{-1} x\right) \nabla u_{\varepsilon} \cdot \nabla \varphi w d \mu_{\varepsilon} & \\
+\varepsilon \lambda \int_{\Omega} u_{\varepsilon} \varphi w d \mu_{\varepsilon} & =\varepsilon \int_{\Omega} f \varphi w d \mu_{\varepsilon} .
\end{aligned}
$$

Все слагаемые, кроме первого, сходятся к нулю. Тогда сходится к нулю и первое, т.е. выполнено условие (4.6). Лемма 4.3 дает слабую сходимость (4.7). Кроме того, имеем $u_{\varepsilon} \rightarrow u, f_{\varepsilon} \rightarrow f$. Поэтому в тождестве (1.13) можно перейти к пределу и получить

$$
\int_{\Omega} A^{\mathrm{hom}} \nabla u \cdot \nabla \psi d x+\lambda \int_{\Omega} u \psi d x=\int_{\Omega} f \psi d x .
$$

Тем самьм доказана первая часть теоремы о слабой сходимости решений.

Остается получить сильную сходимость $u_{\varepsilon} \rightarrow u$ при условии, что правые части сходятся сильно. Это достигается следующим простьм рассуждением. Рассмотрим задачу:

$$
z_{\varepsilon} \in H_{0}^{1}\left(\Omega, d \mu_{\varepsilon}\right), \quad-\operatorname{div}\left(A\left(\varepsilon^{-1} x\right) \nabla z_{\varepsilon}\right)+\lambda z_{\varepsilon}=u_{\varepsilon} .
$$

Так как $u_{\varepsilon} \rightarrow u$, то по доказанному выше $z_{\varepsilon} \rightarrow z$,

$$
z \in H_{0}^{1}(\Omega), \quad-\operatorname{div}\left(A^{\mathrm{hom}} \nabla z\right)+\lambda z=u .
$$

Умножая уравнение (4.9) скалярно на $u_{\varepsilon}$, а исходное уравнение (1.12) на $z_{\varepsilon}$, получаем

$$
\int_{\Omega} u_{\varepsilon}^{2} d \mu_{\varepsilon}=\int_{\Omega} z_{\varepsilon} f_{\varepsilon} d \mu_{\varepsilon}
$$

Отсюда по определению сильной сходимости $f_{\varepsilon} \rightarrow f$ (см. предложение 1.1)

$$
\lim _{\varepsilon \rightarrow 0} \int_{\Omega} u_{\varepsilon}^{2} d \mu_{\varepsilon}=\lim _{\varepsilon \rightarrow 0} \int_{\Omega} z_{\varepsilon} f_{\varepsilon} d \mu_{\varepsilon}=\int_{\Omega} z f d x=\int_{\Omega} u^{2} d x
$$

и сильная сходимость $u_{\varepsilon} \rightarrow u$ установлена. Теорема доказана. 
4.4. Нам понадобится еще одно свойство двухмасштабной сходимости, но сначала введем класс $L^{2}\left(\Omega, H_{\mathrm{per}}^{1}\right)$. Скажем, что функция $u=u(x, y) \in L^{2}(\Omega \times \square)$ принадлежит $L^{2}\left(\Omega, H_{\mathrm{per}}^{1}(\square, d \mu)\right)$ и $\nabla_{y} u \in L^{2}(\Omega \times \square)^{N}$ - ее градиент, если

$$
\begin{gathered}
\exists u_{n} \in C^{\infty}(\bar{\Omega} \times \square): \quad u_{n}-\text { периодичны по } y, \\
u_{n} \rightarrow u, \quad \nabla_{y} u_{n} \rightarrow \nabla_{y} u \quad \text { в } \quad L^{2}(\Omega \times \square) .
\end{gathered}
$$

Из определения видно, что $\nabla_{y} u \in L^{2}\left(\Omega, V_{\text {pot }}\right)$. Если выполнено неравенство Пуанкаре (1.16), то верно и обратное: каждому вектору $v=v(x, y) \in L^{2}\left(\Omega, V_{\text {pot }}\right)$ соответствует единственная функция $\widehat{u}=\widehat{u}(x, y)$ такая, что

$$
\widehat{u}(x, \cdot) \in H_{\mathrm{per}}^{1}, \quad v=\nabla_{y} u, \quad\langle\widehat{u}\rangle=0,
$$

и ясно, что $\widehat{u} \in L^{2}\left(\Omega, H_{\mathrm{per}}^{1}\right)$.

Изучим ситуацию, ког да $u_{\varepsilon} \in C_{0}^{\infty}(\Omega)$ и $u_{\varepsilon}(x), \varepsilon \nabla u_{\varepsilon}(x)$ ограничены в $L^{2}\left(\Omega, d \mu_{\varepsilon}\right)$.

ТЕОРема 4.5. Предположим, что выполнено неравенство Пуанкаре (1.16). Тогда (с точностью до выделения подпоследовательности) имеет место (1.10).

ДокАЗАтЕльство. Возьмем $a \in L^{2}(\square, d \mu), b \in L^{2}(\square, d \mu)^{N}$, связанные равенством $\operatorname{div} b=a$. Пусть $\varepsilon \nabla u_{\varepsilon}(x) \stackrel{2}{\rightarrow} v(x, y)$. Тогда для $\varphi \in C_{0}^{\infty}(\Omega)$

$$
\begin{aligned}
\int_{\Omega} & \varepsilon \nabla u_{\varepsilon}(x) \varphi(x) \cdot b\left(\varepsilon^{-1} x\right) d \mu_{\varepsilon} \\
& =\varepsilon \int_{\Omega} \nabla\left(\varphi u_{\varepsilon}\right) \cdot b\left(\varepsilon^{-1} x\right) d \mu_{\varepsilon}-\varepsilon \int_{\Omega} u_{\varepsilon}(x) \nabla \varphi(x) \cdot b\left(\varepsilon^{-1} y\right) d \mu_{\varepsilon} \\
& =-\int_{\Omega} u_{\varepsilon}(x) \varphi(x) a\left(\varepsilon^{-1} x\right) d \mu_{\varepsilon}-\varepsilon \int_{\Omega} u_{\varepsilon}(x) \nabla \varphi(x) \cdot b\left(\varepsilon^{-1} x\right) d \mu_{\varepsilon} .
\end{aligned}
$$

Отсюда

$$
\begin{aligned}
\int_{\Omega} \int_{\square} \varphi(x) v(x, y) \cdot b(y) d x d \mu & =-\int_{\Omega} \int_{\square} \varphi(x) u(x, y) \operatorname{div} b d x d \mu, \\
\int_{\square} v(x, y) \cdot b(y) d \mu & =-\int_{\square} u(x, y) a(y) d \mu .
\end{aligned}
$$

В частности, полагая $a=0$, видим, что $v \in L^{2}\left(\Omega, V_{\text {pot }}\right)$. Для вектора $v$ рассмотрим потенциал $\widehat{u}$ из (4.10). Тогда из условия $\operatorname{div} b=a$ имеем

$$
\int_{\square} v(x, y) \cdot b(y) d \mu=\int_{\square} \nabla_{y} \widehat{u}(x, y) \cdot b(y) d \mu=-\int_{\square} \widehat{u}(x, y) a(y) d \mu .
$$

Мы получили равенство

$$
\int_{\square} u(x, y) a(y) d \mu=\int_{\square} \widehat{u}(x, y) a(y) d \mu .
$$

Из него по лемме об аппроксимации

$$
\widehat{u}(x, y)=u(x, y)-\int_{\square} u(x, y) d \mu
$$

и поэтому $v=\nabla_{y} \widehat{u}=\nabla_{y} u$. Теорема доказана.

Отметим, что теорема 4.5 была ранее доказана в [19], [20] для случая, когда $\mu$ есть поверхностная мера на гладкой периодической поверхности. 


\section{§5. Усреднение в некоторых моделях пористых сред}

5.1. Сформулируем математическое представление о параллельных потоках, "мягко" или слабо связанных между собой.

Пусть $\mu$ - периодическая борелевская мера в $\mathbb{R}^{N}, F=F_{1} \cup F_{2} \cup \cdots \cup F_{k}$ - объединение периодических $\mu$-измеримых множеств, непересекаюшихся в том смысле, что

i) $\exists E_{i} \in H_{\mathrm{per}}^{1}(\square, d \mu):\left.\nabla E_{i}\right|_{F}=0,\left.E_{i}\right|_{F_{j}}=\delta_{i j}$ - символ Кронекера.

Это условие, очевидно, выполнено, если, например, $\bar{F}_{i} \cap \bar{F}_{j}=0(i \neq j)$.

Мягкую фазу определим как дополнение $F_{0}=\mathbb{R}^{N} \backslash F$.

Зададим проницаемость такой среды матрицей

$$
A_{\varepsilon}(x)= \begin{cases}A_{i}\left(\varepsilon^{-1} x\right), & \text { если } x \in F_{i}^{\varepsilon}, i=1, \ldots, k, \\ \delta(\varepsilon) A_{0}\left(\varepsilon^{-1} x\right), & \text { если } x \in F_{0}^{\varepsilon} .\end{cases}
$$

Здесь $F_{i}^{\varepsilon}=\varepsilon F_{i}$ - гомотетические сжатия множеств $F_{i}, A_{i}(y)-\mu$-измеримые симметрические матрищы, подчиненные условию ограниченности и эллиптичности $(1.2), 0<\delta(\varepsilon) \rightarrow 0$ при $\varepsilon \rightarrow 0$. В физической литературе принято брать $\delta(\varepsilon)=\varepsilon^{2}$. Такая “автомодельная" проницаемость на мягкой фазе действительно приводит к наиболее интересным математическим проблемам. Случаи иного поведения (а их всего два: $\varepsilon^{-2} \delta(\varepsilon) \rightarrow \infty$ и $\varepsilon^{-2} \delta(\varepsilon) \rightarrow 0$ ) исследуются проше. Мы сделаем это в $\S 6$.

Кроме условия і) мы будем предполагать, что

ii) для меры $\mu$ выполнено неравенство Пуанкаре (1.16);

iii) меры $\left.\mu\right|_{F_{i}}(i=1, \ldots, k)$ эргодичны и невырождены.

Отметим, что мы требуем невырожденности мер $\left.\mu\right|_{F_{i}}(i=1, \ldots, k)$ только ради упрощения формулировки; требование эргодичности этих мер - существенно.

На мягкую фазу $F_{0}$ не накладывается никаких условий, кроме $\mu\left(F_{0}\right)>0$.

Рассмотрим задачу Дирихле $(1.12)$, в которой матрица $A_{\varepsilon}(x)$ имеет вид $(5.2)$, а правые части $f_{\varepsilon}$ ограничены в $L^{2}\left(\Omega, d \mu_{\varepsilon}\right)$. Область $\Omega$ распадается на части:

$$
\begin{gathered}
\Omega_{i}^{\varepsilon}=\Omega \cap F_{i}^{\varepsilon}(i=1,2, \ldots, k)-\text { жесткие фазы, } \\
\Omega_{0}^{\varepsilon}=\Omega \cap F_{0}^{\varepsilon}-\text { мягкая фаза. }
\end{gathered}
$$

Из интегрального тождества

$$
\begin{aligned}
\sum_{i=1}^{k} \int_{\Omega_{i}^{\varepsilon}} A_{i}\left(\varepsilon^{-1} x\right) \nabla u_{\varepsilon} \cdot \nabla \psi & d \mu_{\varepsilon}+\delta(\varepsilon) \int_{\Omega_{0}^{\varepsilon}} A_{0}\left(\varepsilon^{-1} x\right) \nabla u_{\varepsilon} \cdot \nabla \psi d \mu_{\varepsilon} \\
& +\lambda \int_{\Omega} u_{\varepsilon} \psi d \mu_{\varepsilon}=\int_{\Omega} f_{\varepsilon} \psi d \mu_{\varepsilon}, \quad \psi \in C_{0}^{\infty}(\Omega),
\end{aligned}
$$

непосредственно следует оценка

$$
\int_{\Omega \backslash \Omega_{0}^{\varepsilon}}\left|\nabla u_{\varepsilon}\right|^{2} d \mu_{\varepsilon}+\delta(\varepsilon) \int_{\Omega_{0}^{\varepsilon}}\left|\nabla u_{\varepsilon}\right|^{2} d \mu_{\varepsilon}+\lambda \int_{\Omega} u_{\varepsilon}^{2} d \mu_{\varepsilon} \leqslant C<\infty .
$$


Наша цель - перейти к пределу в интегральном тождестве и получить предельное уравнение. В первую очередь стоит описать само предельное уравнение.

Пределом решений $u_{\varepsilon}(x)$ будет функция $u(x, y)$ двух переменных $x \in \Omega$ и $y \in \square$, принадлежашая классу $V$, который коротко описьвается так:

$$
\begin{gathered}
u \in L^{2}\left(\Omega, H_{\mathrm{per}}^{1}\right), \\
\left.\nabla_{y} u\right|_{F}=0,\left.\quad u\right|_{F_{j}}=u_{j}(x) \in H_{0}^{1}(\Omega) .
\end{gathered}
$$

Иначе говоря, берутся такие функции $u \in L^{2}\left(\Omega, H_{\mathrm{per}}^{1}\right)$, для которых (хотя бы один) градиент $\nabla_{y} u$ подчинен условию $\nabla_{y} u=0$ в $L^{2}(\Omega \times F)$. Из эргодичности мер $\left.\mu\right|_{F_{i}}(i=1,2, \ldots, k)$ следует, что $\left.u\right|_{F_{i}}$ зависит только от $x \in \Omega$; дополнительно требуется $\left.u\right|_{F_{i}}=u_{i}(x) \in H_{0}^{1}(\Omega)$. Рисунок 5 соответствует случаю двух жестких фаз. Здесь функция $u$ определяется двумя жесткими компонентами $u_{1}(x), u_{2}(x)$ и мягкой компонентой $\left.u\right|_{F_{0}}$, которая зависит от $x \in \Omega$ и $y \in F_{0}$.

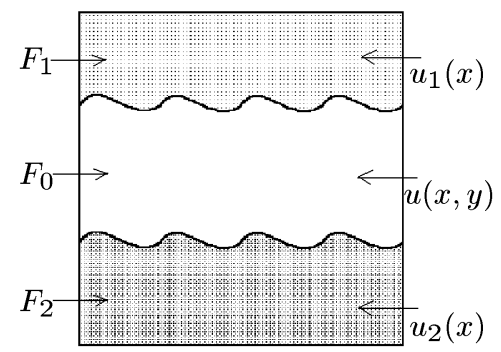

Рис. 5

Полезно записать функцию $u \in V$ в виде

$$
u(x, y)=\sum_{i=1}^{k} u_{i}(x) E_{i}(y)+v(x, y)
$$

где $E_{i}$ - разделяющие функции. Из (3.1), (5.5) видим, что $\left.v\right|_{F}=\left.\nabla_{y} v\right|_{F}=0$. Другими словами, $v \in L^{2}(\Omega, X)$, где мы положили

$$
X=\left\{h \in H_{\mathrm{per}}^{1}(\square, d \mu):\left.h\right|_{F}=\left.\nabla h\right|_{F}=0\right\} .
$$

Пусть $A_{i}^{\text {hom }}$ - усредненные матрицы, соответствующие матрицам $A_{i}$ и мерам $\left.\mu\right|_{F_{i}}$ :

$\xi A_{i}^{\mathrm{hom}} \xi=\inf _{\psi \in C_{\mathrm{per}}^{\infty}(\square)} \int_{\square \cap F_{i}} A_{i}(\xi+\nabla \psi) \cdot(\xi+\nabla \psi) d \mu, \quad A_{i}^{\mathrm{hom}}>0(i=1,2, \ldots, k)$.

Тогда предельная задача формулируется так: для данного $f \in L^{2}(\Omega \times \square)$ найти $u \in V$ такую, что выполнено интегральное тождество

$$
\begin{array}{rl}
\sum_{i=1}^{k} \int_{\Omega} A_{i}^{\text {hom }} \nabla u_{i} \cdot \nabla \varphi_{i} & d x \\
+\int_{\Omega} \int_{\square} A_{0}(y) \nabla_{y} u \cdot \nabla_{y} \varphi d x d \mu \\
+\lambda \int_{\Omega} \int_{\square} u \varphi d x d \mu=\int_{\Omega} \int_{\square} f \varphi d x d \mu, \quad \varphi \in V,
\end{array}
$$

в котором $\nabla_{y} u$ - некоторый градиент и. 
Сушествование и единственность решения как пары $u, \nabla_{y} u$ получается совсем просто. Действительно, левая часть (5.6) задает скалярное произведение на множестве пар $\widetilde{V}=\{u, \nabla u: u \in V\}$ и $\widetilde{V}$ полно относительно соответствующей нормы. Поэтому достаточно сослаться на теорему Рисса.

В дальнейшем используются пробные функции $\varphi \in V$ частного вида

$$
\varphi(x, y)=\sum_{i=1}^{k} \varphi_{i}(x) E_{i}(y)+\alpha(x) h(y), \quad \alpha, \varphi_{1}, \ldots, \varphi_{k} \in C_{0}^{\infty}(\Omega), \quad h \in X .
$$

Легко видеть, что линейная оболочка таких пробных функций (точнее, пар $\varphi$, $\left.\nabla_{y} \varphi\right)$ плотна в $\widetilde{V}$ относительно упомянутой выше нормы. Поэтому само интегральное тождество (5.7) достаточно проверить только для этих пробных функций.

Сформулируем один из результатов работы.

ТЕОрема 5.1. Пусть $\delta(\varepsilon)=\varepsilon^{2}$ и правые части $f_{\varepsilon}$ в (1.12) слабо (сильно) двухмаситабно сходятся $\kappa f \in L^{2}(\Omega \times \square)$. Тогда последовательность решений $u_{\varepsilon}$ слабо (сильно) двухмасштабно сходится к решению задачи (5.7).

Доказательство дано в $\S 6$, а сейчас мы обсудим само предельное уравнение.

5.2. С пространством $X$ будут связаны краевые задачи, описывающие "мягкую” компоненту решения предельного уравнения, и нам потребуется неравенство

$$
\left\langle h^{2}\right\rangle \leqslant C\left\langle|\nabla h|^{2}\right\rangle, \quad h \in X .
$$

Действительно, допустив, что оно не имеет места, найдем

$$
h_{n} \in X: \quad\left\langle h_{n}^{2}\right\rangle=1, \quad\left\langle\left|\nabla h_{n}\right|^{2}\right\rangle \rightarrow 0 .
$$

Тогда из неравенства Пуанкаре (1.16) следует, что

$$
\left\langle\left|h_{n}-c_{n}\right|^{2}\right\rangle \rightarrow 0, \quad c_{n}=\left\langle h_{n}\right\rangle .
$$

Поэтому последовательность $h_{n}$ компактна в $L^{2}(\square, d \mu)$ и ее предел $h$ есть ненулевая константа. В то же время, $\left.h\right|_{F}=0$, что невозможно.

В качестве образца краевой задачи возьмем следующую:

$w \in X, \quad-\operatorname{div}\left(A_{0} \nabla w\right)=p-\operatorname{div} q$ в $F_{0}$, где $p \in L^{2}\left(F_{0}, d \mu\right), q \in L^{2}\left(F_{0}, d \mu\right)^{N}$.

По определению функция $w \in X$ есть решение, если

$$
\left\langle A_{0} \nabla w \cdot \nabla h\right\rangle=\langle p h\rangle+\langle q \cdot \nabla h\rangle \quad \forall h \in X .
$$

С помощью оценки (5.9) нетрудно доказать, что решение сушествует и единственно вместе с градиентом.

Воспользуемся этим фактом и убедимся, что разделяющие функции $E_{j}$ можно подчинить дополнительному условию $\operatorname{div}\left(A_{0} \nabla E_{j}\right)=0$ в $F_{0}$, т.е.

$$
\left\langle A_{0} \nabla E_{j} \cdot \nabla h\right\rangle=0 \quad \forall h \in X .
$$

Достаточно вместо $E_{j}$ взять $E_{j}+v_{j}$, где $v_{j}$ - решение задачи $v_{j} \in X, \operatorname{div} A_{0} \nabla v_{j}$ $=-\operatorname{div}\left(A_{0} \nabla E_{j}\right)$ в $F_{0} . \mathrm{B}$ свою очередь, из (5.11) следует равенство

$$
\sum_{j=1}^{k} \nabla E_{j}=0 \quad \mu \text {-п.в. }
$$

Действительно, $-1+\sum_{j=1}^{k} E_{j}=v \in X$ и $\operatorname{div} A_{0} \nabla v=0$ в $F_{0}$. Поэтому $\nabla v \equiv 0$. 
5.3. Сейчас мы выведем некоторые свойства решения предельной задачи (5.7). При этом будем использовать только пробные функции вида (5.8).

Возьмем в тождестве (5.7) пробную функцию $\varphi=\varphi_{1}(x) E_{1}(y)$. Тогда

$$
\begin{aligned}
\int_{\Omega} A_{1}^{\text {hom }} \nabla u_{1} \cdot \nabla \varphi_{1} d x & +\int_{\Omega} \int_{\square} A_{0} \nabla_{y} u \cdot \nabla E_{1} \varphi_{1} d \mu d x \\
& +\lambda \int_{\Omega} \int_{\square} u E_{1} \varphi_{1} d \mu d x=\int_{\Omega} \int_{\square} f E_{1} \varphi_{1} d \mu d x .
\end{aligned}
$$

Аналогично, взяв $\varphi=\varphi_{i}(x) E_{i}(y)$, придем к системе

$$
\left\{\begin{array}{l}
-\operatorname{div}\left(A_{1}^{\mathrm{hom}} \nabla u_{1}\right)+\left\langle A_{0} \nabla_{y} u \cdot \nabla E_{1}\right\rangle+\lambda\left\langle u E_{1}\right\rangle=\left\langle f E_{1}\right\rangle \\
\ldots \ldots \ldots \ldots \ldots \ldots \ldots \ldots \ldots \ldots \ldots \ldots \ldots \ldots \ldots \ldots \ldots \ldots \ldots \ldots \ldots \ldots \ldots \ldots \ldots \ldots \ldots \\
-\operatorname{div}\left(A_{k}^{\mathrm{hom}} \nabla u_{k}\right)+\left\langle A_{0} \nabla_{y} u \cdot \nabla E_{k}\right\rangle+\lambda\left\langle u E_{k}\right\rangle=\left\langle f E_{k}\right\rangle .
\end{array}\right.
$$

Далее, положим в тождестве $(5.7) \varphi=\alpha(x) h(y)$. Тогда получим

$$
\left\{\begin{array}{c}
-\operatorname{div}_{y}\left(A_{0}(y) \nabla_{y} u\right)+\lambda u=f \text { в } F_{0}, \\
\left.u\right|_{F_{i}}=u_{i}(x) .
\end{array}\right.
$$

Это эллиптическая по $y$ задача, в которой $x$ играет роль параметра. Из нее можно выразить $u(x, y)$ через компоненты $u_{i}(x)$ и получить для $u_{i}$ связанную систему уравнений. Для этого введем решения следующих эллиптических задач:

$$
\begin{gathered}
b_{i}-E_{i} \in X, \quad-\operatorname{div}_{y}\left(A_{0}(y) \nabla_{y} b_{i}\right)+\lambda b_{i}=0 \text { в } F_{0} \quad(i=1,2, \ldots, k), \\
b_{0} \in L^{2}(\Omega, X), \quad-\operatorname{div}_{y}\left(A_{0} \nabla_{y} b_{0}\right)+\lambda b_{0}=f \text { в } F_{0} .
\end{gathered}
$$

Тогда имеем

$$
\begin{gathered}
u(x, y)=b_{0}(x, y)+\sum_{j=1}^{k} u_{j}(x) b_{j}(y) \\
\left\langle A_{0} \nabla_{y} u \cdot \nabla E_{i}\right\rangle=\left\langle A_{0}\left(u_{1} \nabla b_{1}+\cdots+u_{k} \nabla b_{k}+\nabla_{y} b_{0}\right) \cdot \nabla E_{i}\right\rangle .
\end{gathered}
$$

Поскольку $\left\langle A_{0} \nabla_{y} b_{0} \cdot \nabla E_{i}\right\rangle=0$ в силу (5.11), то получаем систему

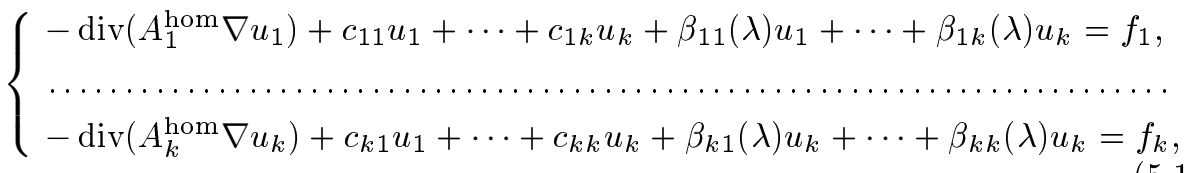

в которой

$$
\begin{gathered}
c_{i j}=\left\langle A_{0} \nabla E_{i} \cdot \nabla b_{j}\right\rangle, \quad \beta_{i j}=\lambda\left\langle E_{i} b_{j}\right\rangle \\
f_{j}(x, \lambda)=\left\langle\left(f-\lambda b_{0}\right) E_{j}\right\rangle \quad(i, j=1,2, \ldots, k) .
\end{gathered}
$$


Так как $b_{i}-E_{i} \in X$, то

$$
c_{i j}=\left\langle A_{0} \nabla E_{i} \cdot \nabla b_{j}\right\rangle=\left\langle A_{0} \nabla E_{i} \cdot \nabla E_{j}\right\rangle=c_{j i} \text { (симметрия). }
$$

Заметим, что матрица $\left\{c_{i j}\right\}$ - неотрицательно определенная и не зависит от $\lambda$. Эта "матрица связи" удовлетворяет условию $\sum_{i} c_{i j}=0$, как это следует из (5.12). Для $k=2$ матрица связи имеет структуру

$$
\left(\begin{array}{cc}
q & -q \\
-q & q
\end{array}\right), \quad q=\left\langle A_{0} \nabla E_{1} \cdot \nabla E_{1}\right\rangle
$$

Нетрудно проверить, что матрица $\left\{\beta_{i j}\right\}$ - также симметрическая и положительно определенная. Нелинейный характер ее зависимости от $\lambda$ указывает, что система (5.15) как таковая не является резольвентной. В этом нет ничего особенного, поскольку эта система - всего лишь некоторая "проекция" предельной задачи (5.7). Сама же предельная задача имеет резольвентный характер, и это - наиболее важное в данном случае обстоятельство.

\section{§6. Доказательство сходимости}

6.1. Будем изучать общий случай задания проницаемости на мягкой фазе: $\delta(\varepsilon) \rightarrow 0$ произвольно, см. (5.2).

Пусть $u_{\varepsilon}$ - решение исходной задачи (1.12). Без потери общности считаем, что имеет место слабая двухмасштабная сходимость

$$
u_{\varepsilon}(x) \stackrel{2}{\rightarrow} u(x, y)
$$

Нам нужно выяснить структуру предельной функции $u$.

Лемма 6.1. Найдутся функции $u_{1}(x), u_{2}(x), \ldots, u_{k}(x) \in H_{0}^{1}(\Omega)$ такие, что

$$
\begin{gathered}
u(x, y)=u_{1}(x), \quad \text { ecлu } \quad y \in F_{1}, \\
\lim _{\varepsilon \rightarrow 0} \int_{\Omega_{1}^{\varepsilon}} A_{1}\left(\varepsilon^{-1} x\right) \nabla u_{\varepsilon} \cdot \nabla \varphi d \mu_{\varepsilon}=\int_{\Omega} A_{1}^{\mathrm{hom}} \nabla u_{1} \cdot \nabla \varphi d x \quad \forall \varphi \in C_{0}^{\infty}(\Omega)
\end{gathered}
$$

и аналогично для других жестких компонент.

ДокаЗАТЕЛЬСтво. Пусть $\chi_{1}(y)$ - характеристическая функция множества $F_{1}$. Тогда по свойству (2.7) двухмасштабной сходимости

$$
\chi_{1}\left(\varepsilon^{-1} x\right) u_{\varepsilon}(x) \stackrel{2}{\rightarrow} \chi_{1}(y) u(x, y) .
$$

$\mathrm{C}$ другой стороны, сужения $\left.u_{\varepsilon}\right|_{\Omega_{1}^{\varepsilon}},\left.\nabla u_{\varepsilon}\right|_{\Omega_{1}^{\varepsilon}}$ удовлетворяют условиям теоремы 4.2 по отношению к мере $\left.\mu\right|_{F_{1}}$. Поэтому $\chi_{1}\left(\varepsilon^{-1} x\right) u_{\varepsilon}(x) \stackrel{2}{\rightarrow} u_{1}(x) \in H_{0}^{1}(\Omega)$ и равенство (6.2) доказано. Далее, для пробной функции

$$
\psi(x)=\psi_{\varepsilon}(x)=\varepsilon w(y) \varphi(x), \text { где } y=\varepsilon^{-1} x, w(y) \in C_{\mathrm{per}}^{\infty}(\square), \varphi(x) \in C_{0}^{\infty}(\Omega),
$$


имеем

$\left.\nabla \psi_{\varepsilon}\right|_{\Omega_{1}^{\varepsilon}}=\varphi(x) \nabla_{y} w+\varepsilon w \nabla \varphi,\left.\quad \nabla \psi_{\varepsilon}\right|_{\Omega_{j}^{\varepsilon}}=\varepsilon w \nabla \varphi$ при $j>1, \quad\left|\nabla \psi_{\varepsilon}\right| \leqslant C$ на $\Omega_{0}^{\varepsilon}$.

Подставим $\psi=\psi_{\varepsilon}$ в интегральное тождество (5.3). Из оценок (5.4) легко заключаем, что все слагаемые, кроме, может быть, слагаемого

$$
\int_{\Omega_{1}^{\varepsilon}} A_{1}\left(\varepsilon^{-1} x\right) \nabla u_{\varepsilon}(x) \cdot \nabla_{y} w\left(\varepsilon^{-1} x\right) \varphi(x) d \mu_{\varepsilon}
$$

сходятся к нулю при $\varepsilon \rightarrow 0$. Но тогда и указанное слагаемое сходится к нулю. По лемме 4.3 получаем искомую сходимость (6.3), и доказательство закончено.

Доказанная лемма описывает поведение решений $u_{\varepsilon}$ на жестких компонентах. Поведение решений на мягкой компоненте уже зависит от асимптотики $\delta(\varepsilon)$.

6.2. Случай $\delta(\varepsilon)=\varepsilon^{2}$. Этот случай соответствует сформулированной ранее теореме 5.1, которую мы сейчас докажем. Здесь $u_{\varepsilon}, \varepsilon \nabla u_{\varepsilon}$ ограничены в $L^{2}\left(\Omega, d \mu_{\varepsilon}\right)$ и по теореме $4.5 u(x, y) \in L^{2}\left(\Omega, H_{\mathrm{per}}^{1}\right), \varepsilon \nabla u_{\varepsilon} \stackrel{2}{\rightarrow} \nabla_{y} u$. Поскольку $\varepsilon \chi_{j}\left(\varepsilon^{-1} x\right) \nabla u_{\varepsilon} \rightarrow 0$ в $L^{2}\left(\Omega, d \mu_{\varepsilon}\right)(j=1,2, \ldots, k)$, то $\left.\nabla_{y} u\right|_{F}=0$. Поэтому функция $u$ принадлежит классу $V$, см. (5.5).

Положим $\psi(x)=\psi_{\varepsilon}(x)=\varphi\left(x, \varepsilon^{-1} x\right)$, где $\varphi$ - пробная функция (5.8). Очевидно,

$$
\left.\nabla \psi_{\varepsilon}\right|_{\Omega_{j}^{\varepsilon}}=\nabla \varphi_{j}, \quad \varepsilon \nabla \psi_{\varepsilon}=\nabla_{y} \varphi+o(1) \text { по норме } L^{2}\left(\Omega, d \mu_{\varepsilon}\right) .
$$

Подставим $\psi=\psi_{\varepsilon}$ в интегральное тождество (5.3):

$$
\begin{aligned}
& \sum_{j=1}^{k} \int_{\Omega_{j}^{\varepsilon}} A_{j}\left(\varepsilon^{-1} x\right) \nabla u_{\varepsilon}(x) \cdot \nabla \varphi_{j}(x) d \mu_{\varepsilon} \\
&+\delta(\varepsilon) \varepsilon^{-1} \int_{\Omega_{0}^{\varepsilon}} A_{0}\left(\varepsilon^{-1} x\right) \nabla u_{\varepsilon} \cdot\left[\nabla_{y} \varphi\left(x, \varepsilon^{-1} x\right)+o(1)\right] d \mu_{\varepsilon} \\
&+\lambda \int_{\Omega} u_{\varepsilon} \varphi d \mu_{\varepsilon}=\int_{\Omega} f_{\varepsilon} \varphi d \mu_{\varepsilon}
\end{aligned}
$$

Перейдем здесь к пределу при $\varepsilon \rightarrow 0$. Предел первой суммы уже вычислен в (6.3). Далее, в нашем случае $\delta(\varepsilon) \varepsilon^{-1}=\varepsilon$, и поэтому предел второго слагаемого, вычисляемый по двухмасштабной сходимости

$$
\varepsilon A_{0}\left(\varepsilon^{-1} x\right) \nabla u_{\varepsilon}(x) \stackrel{2}{\rightarrow} A_{0}(y) \nabla_{y} u(x, y),
$$

равен $\int_{\square} \int_{\Omega} A_{0} \nabla_{y} u \cdot \nabla_{y} \varphi d x d \mu$. В результате получим, что $u$ удовлетворяет тождеству (5.7) на пробных функциях из (5.8). Как уже отмечалось, этого достаточно для того, чтобы $u$ было решением предельной задачи (5.7).

Нам остается проверить, что имеет место сильная сходимость $u_{\varepsilon}(x) \stackrel{2}{\rightarrow} u(x, y)$, если только правые части также сходятся сильно. Но это достигается тем же рассуждением, что и в теореме 4.4. Теорема 5.1 доказана. 
6.3. Случай $\varepsilon^{-2} \delta(\varepsilon) \rightarrow \infty$. Здесь $\varepsilon\left\|\nabla u_{\varepsilon}\right\|_{L^{2}\left(\Omega, d \mu_{\varepsilon}\right)} \rightarrow 0$. Поэтому предельная функция $u$ не только принадлежит $V$, но и вовсе не зависит от $y: u(x, y)=u(x)$, в частности $u_{1}=u_{2}=\cdots=u_{k}$. Посмотрим, как перейти к пределу в тождестве (5.3). Предел первой суммы слева вычисляется по (6.3). Далее заметим, что

$$
\delta(\varepsilon) \int_{\Omega_{0}^{\varepsilon}} A_{0}\left(\varepsilon^{-1} x\right) \nabla u_{\varepsilon}(x) \cdot \nabla \psi(x) d \mu_{\varepsilon} \rightarrow 0
$$

как это ясно из оценки $\delta(\varepsilon) \int_{\Omega_{0}^{\varepsilon}}\left|\nabla u_{\varepsilon}\right|^{2} d \mu_{\varepsilon} \leqslant C<\infty$. Считая, что $f_{\varepsilon} \rightarrow f \in L^{2}(\Omega)$, в пределе получим

$$
\begin{gathered}
u \in H_{0}^{1}(\Omega),-\operatorname{div}\left(A^{\mathrm{hom}} \nabla u\right)+\lambda u=f, \\
A^{\text {hom }}=A_{1}^{\mathrm{hom}}+A_{2}^{\mathrm{hom}}+\cdots+A_{k}^{\mathrm{hom}} .
\end{gathered}
$$

Мы видим, что предельное уравнение имеет обычный характер.

Теорема 6.2. Пусть $\lim _{\varepsilon \rightarrow 0} \varepsilon^{2} \delta(\varepsilon)=\infty$ и правые части $f_{\varepsilon}$ слабо (сильно) сходятся $\kappa f \in L^{2}(\Omega)$. Тогда последовательность решений слабо (сильно) сходится к решению предельного уравнения (6.5).

Видим, что формулировка - в точности такая же, как для классического усреднения (теорема 4.4).

6.4. Случай $\varepsilon^{-2} \delta(\varepsilon) \rightarrow 0$. Здесь нам требуется найти сужение $\left.u\right|_{F_{0}}=u_{0}(x, y)$, где $u(x, y)$ - предельная функция из (6.1). Для этого рассмотрим второе слагаемое в левой части тождества (6.4). В нашем случае оно сходится к нулю:

$$
\delta(\varepsilon) \varepsilon^{-1} \int_{\Omega_{0}^{\varepsilon}} A_{0}\left(\varepsilon^{-1} x\right) \nabla u_{\varepsilon}(x) \cdot \nabla_{y} \varphi\left(x, \varepsilon^{-1} x\right) d \mu_{\varepsilon} \rightarrow 0,
$$

как это ясно из оценки $\delta(\varepsilon) \int_{\Omega_{0}^{\varepsilon}}\left|\nabla u_{\varepsilon}\right|^{2} d \mu_{\varepsilon} \leqslant C$ и неравенства Коши-Буняковского. Если $f_{\varepsilon} \stackrel{2}{\rightarrow} f \in L^{2}(\Omega \times \square)$, то предельный переход в (6.4) дает:

$$
\sum_{j=1}^{k} \int_{\Omega} A_{j}^{\mathrm{hom}} \nabla u_{j} \cdot \nabla \varphi_{j} d x+\lambda \int_{\Omega} \int_{\square} u(x, y) \varphi(x, y) d x d \mu=\int_{\Omega} \int_{\square} f \varphi d x d \mu,
$$

где $\varphi$ - пробная функция из (5.8). Взяв здесь сначала $\varphi=\alpha(x) h(y)$, а затем $\varphi=$ $\sum_{j=1}^{k} \varphi_{j}(x) E_{j}(y)$, найдем, что

$$
\left\{\begin{array}{c}
\lambda u_{0}=\left.f\right|_{F_{0}}, \\
-\operatorname{div}\left(A_{1}^{\mathrm{hom}} \nabla u_{1}\right)+\lambda\left\langle u E_{1}\right\rangle=\left\langle f E_{1}\right\rangle \\
\ldots \ldots \ldots \ldots \ldots \ldots \ldots \ldots \ldots \ldots \ldots \ldots \ldots \ldots \ldots \\
-\operatorname{div}\left(A_{k}^{\mathrm{hom}} \nabla u_{k}\right)+\lambda\left\langle u E_{k}\right\rangle=\left\langle f E_{k}\right\rangle .
\end{array}\right.
$$

Очевидно, эта система полностью распадается и имеет вид

$$
\left\{\begin{array}{c}
\lambda u_{0}=\left.f\right|_{F_{0}}, \\
-\operatorname{div}\left(A_{1}^{\mathrm{hom}} \nabla u_{1}\right)+\lambda \theta_{1} u_{1}=\left\langle\chi_{1} f\right\rangle, \\
\ldots \ldots \ldots \ldots \ldots \ldots \ldots \ldots \ldots \ldots \ldots \ldots \ldots \ldots \ldots \\
-\operatorname{div}\left(A_{k}^{\text {hom }} \nabla u_{k}\right)+\lambda \theta_{k} u_{k}=\left\langle\chi_{k} f\right\rangle,
\end{array}\right.
$$

где $\chi_{i}$ - характеристическая функция множества $F_{i}, \theta_{i}=\mu\left(F_{i}\right)(i=1,2, \ldots, k)$. 
ТЕОРема 6.3. Пусть $\varepsilon^{-2} \delta(\varepsilon) \rightarrow 0$ и правые части $f_{\varepsilon}$ слабо (сильно) двухмасштабно сходятся $\kappa f \in L^{2}(\Omega \times \square)$. Тогда последовательность решений слабо (сильно) двухмасштабно сходится к решению системь (6.6).

\section{§7. Аналог сильной резольвентной сходимости}

7.1. Определим гильбертово пространство $H$ как подпространство в $L^{2}(\Omega \times \square)$, состоящее из функций

$$
f=f(x, y)= \begin{cases}f_{i}(x), & \text { если } y \in F_{i}(i=1, \ldots, k), \\ f_{0}(x, y), & \text { если } y \in F_{0} .\end{cases}
$$

Скалярное произведение в $H$ наследуется из $L^{2}(\Omega \times \square)$. Такой выбор пространства $H$ объясняется очень просто: $H$ - это замыкание множества $V$ в $L^{2}(\Omega \times \square)$, см. (5.5). С предельной задачей (5.7) свяжем самосопряженньй оператор $\mathscr{A}$ так, что само это уравнение запишется как операторное уравнение (3.5) в $H$. Для этого через $D(\mathscr{A})$ обозначим совокупность решений задачи $(5.7)$, в которой $f$ пробегает $H$. На $D(\mathscr{A}) \subset V \subset H$ положим $\mathscr{A} u=f-\lambda u$. Как и в случае уравнения (3.2), нужно проверить, что $D(\mathscr{A})$ плотно в $H$. Для доказательства плотности возьмем второй экземпляр задачи (5.7), в котором $f$ заменено на $g$, а $u$ заменено на $v$. Получаем обычное равенство

$$
\int_{\Omega} \int_{\square} f v d x d \mu=\int_{\Omega} \int_{\square} g u d x d \mu .
$$

Пусть $f \in H, f \perp D(\mathscr{A})$ в $H$. Тогда $u=0$ (поскольку можно взять $g=u$ ) и интегральное тождество (5.7) принимает вид

$$
\int_{\Omega} \int_{\square} A_{0} \nabla_{y} u \cdot \nabla_{y} \varphi d x d \mu=\int_{\Omega} \int_{\square} f \varphi d x d \mu \quad \forall \varphi \in V .
$$

Полагая здесь $\varphi=u$, получаем $\nabla_{y} u=0$. Тогда и $f=0$ ввиду плотности $V$ в $H$. Тем самым доказана самосопряженность оператора $\mathscr{A}$.

Итак, имеем допредельные самосопряжсенные операторьи $\mathscr{A}_{\varepsilon}=-\operatorname{div}\left(A_{\varepsilon} \nabla\right)$, заданные в $L^{2}\left(\Omega, d \mu_{\varepsilon}\right)$, и предельный самосопряжсенный оператор $\mathscr{A}$, заданныци $\boldsymbol{в} H$.

Из теоремы 5.1 получаем следующий результат.

Имеет место сильная резольвентная сходимость, именно:

$$
\text { если } f_{\varepsilon} \stackrel{2}{\rightarrow} f \in H, \quad \text { mo }\left(\mathscr{A}_{\varepsilon}+\lambda I\right)^{-1} f_{\varepsilon} \stackrel{2}{\rightarrow}(\mathscr{A}+\lambda I)^{-1} f .
$$

Указанная сходимость обладает всеми достоинствами классической сильной резольвентной сходимости: она влечет сильную двухмасштабную сходимость соответствующих спектральных проекторов и полугрупп (аналог теоремы Троттера-Като), см. [21]. Эти факты могут быть полезны в теории пористых сред. Дело в том, что исходным объектом в этой теории служит эволюционное уравнение

$$
\frac{\partial u_{\varepsilon}}{\partial t}=\operatorname{div}\left(A_{\varepsilon}(x) \nabla u_{\varepsilon}\right),\left.\quad u_{\varepsilon}\right|_{\partial \Omega}=0,\left.\quad u_{\varepsilon}\right|_{t=0}=f_{\varepsilon} .
$$

Кроме того, важнейшим считается вопрос о характере спектра допредельного оператора $\mathscr{A}_{\varepsilon}$ при малых $\varepsilon$. Сильная сходимость спектральных проекторов позволяет делать на этот счет некоторые заключения, если, конечно, известен спектр предельного оператора. Ниже мы рассматриваем эти вопросы подробнее. 
7.2. Рассмотрим эволюционное уравнение (7.2). По теореме Троттера-Като получаем, что справедлива

ТеОРема 7.1. Пусть начальные значения $f_{\varepsilon}$ сильно двухмаситабно сходятся $\kappa f \in H$. Тогда при любом $t \geqslant 0$

$$
u_{\varepsilon}(x, t) \stackrel{2}{\rightarrow} u(x, y, t),
$$

где $u(x, y, t)$ - решение предельного уравнения

$$
\frac{\partial u}{\partial t}+\mathscr{A} u=0,\left.\quad u\right|_{t=0}=f
$$

Поскольку $\mathscr{A}$ есть самосопряженньй неотрицательный оператор в гильбертовом пространстве $H$, то точный смысл самого предельного уравнения вполне очевиден: для любого начального значения $f \in H$ существует единственная функция $u(t)=e^{-\mathscr{A} t} f$ со значением в $H$ такая, что $u(t)$ непрерывна в $H$ и $u(0)=f$, $u(t)$ дифференцируема при $t>0, u(t) \in D(\mathscr{A}) \subseteq V$ и $u^{\prime}(t)+\mathscr{A} u=0$.

Из стационарных уравнений (5.13), (5.14), полагая $f=-\frac{\partial u}{\partial t}, \lambda=0$, имеем

$$
\begin{aligned}
& \left\{\begin{array}{l}
\frac{\partial}{\partial t}\left\langle u E_{1}\right\rangle-\operatorname{div}\left(A_{1}^{\mathrm{hom}} \nabla u_{1}\right)+\left\langle A_{0} \nabla_{y} u \cdot \nabla E_{1}\right\rangle=0, \\
\cdots \cdots \cdots \cdots \cdots \cdots \cdots \cdots \cdots \cdots \cdots \cdots \cdots \cdots \cdots \cdots \cdots \cdots \cdots \cdots \cdots \cdots \cdots \cdots \cdots \cdots \\
\frac{\partial}{\partial t}\left\langle u E_{k}\right\rangle-\operatorname{div}\left(A_{k}^{\mathrm{hom}} \nabla u_{k}\right)+\left\langle A_{0} \nabla_{y} u \cdot \nabla E_{k}\right\rangle=0,
\end{array}\right. \\
& \frac{\partial u}{\partial t}-\operatorname{div}_{y} A_{0} \nabla_{y} u=0 \text { в } F_{0}, \\
& \left.u\right|_{F_{i}}=u_{i}(x, t)(i=1,2, \ldots, k),\left.\quad u\right|_{t=0}=f .
\end{aligned}
$$

Из последнего уравнения можно выразить решение $u(x, y, t)$ через компоненты $u_{1}(x, t), u_{2}(x, t), \ldots, u_{k}(x, t)$ и получить для $u_{1}, u_{2}, \ldots, u_{k}$ связанную систему с памятью. На подробностях останавливаться не будем, так как они вполне аналогичны случаю резольвентного уравнения. Отметим только, что связанные системы с памятью, по-видимому, впервые были обнаружены в работах Хруслова [22]-[24].

\section{§ 8. О спектре предельного оператора}

8.1. Будем изучать случай, когда жесткая фаза - одна: $k=1, F=F_{1}$. Функцию $u \in V$ запишем в виде

$$
u(x, y)=u_{1}(x)+v(x, y), \quad v \in L^{2}\left(\Omega, H_{\mathrm{per}}^{1}\right),\left.\quad v\right|_{F_{1}}=\left.\nabla_{y} v\right|_{F_{1}}=0,
$$

или короче: $v \in L^{2}(\Omega, X)$ (см. обозначение (5.6)).

Рассмотрим равенство $\mathscr{A} u=f$, где $u \in V, f \in H$. По определению должно вьполняться тождество

$$
\int_{\Omega} A_{1}^{\text {hom }} \nabla u_{1} \cdot \nabla \varphi_{1} d x+\int_{\Omega} \int_{\square} A_{0} \nabla_{y} v \cdot \nabla_{y} \varphi d x d \mu=\int_{\Omega} \int_{\square} f \varphi d x d \mu, \quad \varphi \in V .
$$


Выбирая здесь пробные функции (5.8) частного вида $\varphi=\alpha(x) h(y)$, а затем $\varphi=$ $\varphi_{1}(x)$, получаем два равенства

$$
\left\{\begin{array}{c}
-\operatorname{div}_{y}\left(A_{0}(y) \nabla_{y} v\right)=f(x, y) \text { в } F_{0} \\
-\operatorname{div}\left(A_{1}^{\mathrm{hom}} \nabla u_{1}\right)=\int_{\square} f(x, y) d \mu(y)=\langle f\rangle \text { в } \Omega,
\end{array}\right.
$$

причем в первом из них $x$ играет роль параметра.

Введем два самосопряженных оператора.

i) $\mathscr{A}_{0}=-\operatorname{div}_{y}\left(A_{0}(y) \nabla_{y}\right)$ определен в $L^{2}\left(F_{0}, d \mu\right)$ по следуюшему правилу. Мы знаем (см. (5.10)), что задача

$$
w \in X, \quad-\operatorname{div}_{y}\left(A_{0}(y) \nabla_{y} w\right)=p
$$

имеет решение для любого $p \in L^{2}\left(F_{0}, d \mu\right)$. Оператор $\mathscr{A}_{0}$ задается на множестве решений этого уравнения и оказывается положительно определенным в силу оценки (5.9). Кроме того, мы предполагаем, что обратный к нему вполне непрерывен. В модельных примерах это имеет место.

Тем же символом $\mathscr{A}_{0}$ обозначается и соответствующий оператор в $L^{2}\left(\Omega \times F_{0}\right)$; очевидно, спектр остается тем же, а собственные функции умножаются на произвольную функцию $a(x) \in L^{2}(\Omega)$.

Особенно просто выплядит оператор $\mathscr{A}_{0}$ в том случае, когда $d \mu=d x$ - мера Лебега, а мягкая фаза $F_{0}$ дисперсна, например является периодической системой шаров, $\square \cap F_{0}=B$-шар. Тогда пространство $X$ (см. определение (5.6)) совпадает с множеством функций из $H_{0}^{1}(B)$, продолженных нулем на тор периодичности $\square$. Также с точностью до очевидного продолжения $\mathscr{A}_{0}$ - это самосопряженный оператор $-\operatorname{div}_{y} A_{0}(y) \nabla_{y}$ в $L^{2}(B)$, отвечающий задаче Дирихле в шаре $B$.

ii) $\mathscr{A}_{1}=-\operatorname{div}\left(A_{1}^{\mathrm{hom}} \nabla\right)$ - это обычный усредненный оператор в $L^{2}(\Omega)$, отвечающий краевой задаче Дирихле.

Оператор $\mathscr{A}_{0}$ назовем блоховским, а оператор $\mathscr{A}_{1}$ - пространственнылм.

Теперь рассмотрим задачу на "спектре":

$$
\mathscr{A} u=s u+f, \quad s \geqslant 0 .
$$

Согласно (8.1) имеем

$$
\left\{\begin{aligned}
\mathscr{A}_{0} v & =s\left(u_{1}+v\right)+f, \\
\mathscr{A}_{1} u_{1} & =s\left\langle u_{1}+v\right\rangle+\langle f\rangle .
\end{aligned}\right.
$$

Введем (пока формально) решения вспомогательных уравнений

$$
\mathscr{A}_{0} b=s b+1, \quad \mathscr{A}_{0} b_{0}=s b_{0}+f
$$

Тогда $v=s u_{1} b+b_{0}$,

$$
\mathscr{A}_{1} u_{1}=\beta(s) u_{1}+\left\langle s b_{0}+f\right\rangle
$$

где $\beta(s)=s(1+s\langle b\rangle)$. Задачи (8.3), (8.4) разрешимы, если $s$ не является точкой спектра оператора $\mathscr{A}_{0}$, а $\beta(s)$ - точкой спектра оператора $\mathscr{A}_{1}$. Для таких $s$ уравнение (8.2) имеет решение при любом $f \in H$. Итак,

если $s \notin \mathrm{Sp} \mathscr{A}_{0}$ и $\beta(s) \notin \mathrm{Sp} \mathscr{A}_{1}$, то $s \notin \mathrm{Sp} \mathscr{A}$. 
Изучим подробней спектр оператора $\mathscr{A}$. Рассмотрим задачу на собственные значения

$$
\mathscr{A} u=s u \text { или }\left\{\begin{aligned}
\mathscr{A}_{0} v & =s\left(u_{1}+v\right), \\
\mathscr{A}_{1} u_{1} & =s\left\langle u_{1}+v\right\rangle .
\end{aligned}\right.
$$

Замечаем, что если $\mathscr{A}_{0} v=s v$ и $\langle v\rangle=0$, то оба уравнения (8.5) выполняются при $u_{1}=0$. Другими словами, в этом случае $u=v(y)$ будет собственной функцией оператора $\mathscr{A}$. Собственной функцией будет также $a(x) v(y), a(x) \in L^{2}(\Omega)$. Это тривиальные или чисто блоховские собственные функции.

По условию спектр оператора $\mathscr{A}_{0}$ состоит из конечнократных собственных значений, образующих сходящуюся к бесконечности последовательность положительных чисел. Разобьем его на две непересекающиеся части

$$
\operatorname{Sp} \mathscr{A}_{0}=\left\{\omega_{1}, \omega_{2}, \ldots\right\} \cup\left\{\alpha_{1}, \alpha_{2}, \ldots\right\}
$$

отнеся ко второй части собственные значения, у которых все соответствующие собственные функции имеют нулевое среднее.

Напишем разложение

$$
1=\sum_{n=1}^{\infty} c_{n} \varphi_{n}, \quad c_{n}=\left\langle\varphi_{n}\right\rangle
$$

где $\varphi_{n}$ - нормированные в $L^{2}\left(F_{0}, d \mu\right)$ собственные функции оператора $\mathscr{A}_{0}$. Считаем, что в этом разложении коэффициенты $c_{n} \neq 0$. Другими словами, собственные функции с нулевым средним здесь не участвуют. Тогда из $\mathscr{A}_{0} b=s b+1$ находим

$$
b=b(y, s)=\sum_{n=1}^{\infty} \frac{c_{n} \varphi_{n}(y)}{\omega_{n}-s}, \quad \beta(s)=s(1+s\langle b\rangle)=s+\sum_{n=1}^{\infty} \frac{s^{2} c_{n}^{2}}{\omega_{n}-s} .
$$

Первое из уравнений (8.5) выполняется, если положить $v(x, y)=s u_{1}(x) b(y, s)$. Поскольку $s\left\langle u_{1}+v\right\rangle=\beta(s) u_{1}(x)$, то второе уравнение удовлетворяется, если $k=\beta(s)$ есть точка спектра оператора $\mathscr{A}_{1}$, а $u_{1}(x)$ - соответствующая собственная функция.

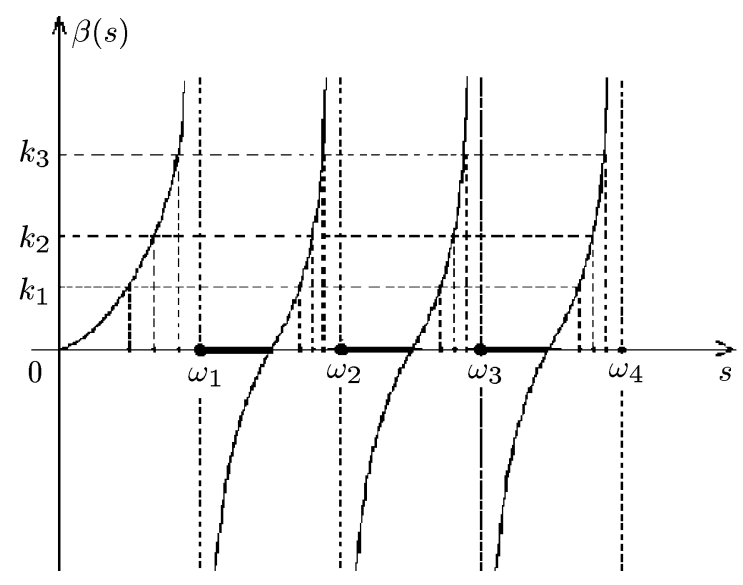

Рис. 6 
Функция $\beta(s)$ строго возрастает на промежутках между точками $0, \omega_{1}, \omega_{2}, \ldots$. Из рис. 6 видно, что решения уравнения $\beta(s)=k \in \mathrm{Sp} \mathscr{A}_{1}$ образуют бесконечные серии, имеющие своими пределами точки $\omega_{1}, \omega_{2}, \ldots$ В результате получим:

если $k=\beta(s) \in \mathrm{Sp} \mathscr{A}_{1}, u_{1}(x)$ - собственная функиия оператора $\mathscr{A}_{1}$ с собственным значением $k$, то $u_{1}(x)(1+s b(y))$ - собственная функция оператора $\mathscr{A}$ с собственным значением $s$.

Найденные "пространственные" собственные значения, конечно, могут попасть в множество $\left\{\alpha_{1}, \alpha_{2}, \ldots\right\}$, и тогда им будут соответствовать еще и чисто блоховские собственные функции. Покажем, что никаких других собственных функиий (кроме указанных в (8.6) и возможных блоховских) нет. Для этого достаточно проверить, что уравнение (8.2) разрешимо, если $f$ ортогонально в $L^{2}(\Omega \times \square)$ собственным функциям из (8.6) и соответствующим блоховским, т.е.

$$
\begin{gathered}
\int_{\Omega} \int_{\square} f(x, y) u_{1}(x)(1+s b(y)) d x d \mu=0 \\
\int_{\Omega} \int_{\square} f(x, y) a(x) \varphi(y) d x d \mu=0, \text { как только } \mathscr{A}_{0} \varphi=s \varphi,\langle\varphi\rangle=0, a \in L^{2}(\Omega) .
\end{gathered}
$$

Первое из уравнений (8.3), очевидно, разрешимо, поскольку $s \notin\left\{\omega_{1}, \omega_{2}, \ldots\right\}$. Разрешимость второго следует из (8.8). Для разрешимости уравнения (8.4) нужно проверить условие ортогональности

$$
\left\langle s b_{0}+f\right\rangle \perp u_{1} \quad \text { в } \quad L^{2}(\Omega) .
$$

Но из уравнений (8.3) имеем равенство $\left\langle b_{0}\right\rangle=\langle f b\rangle$, откуда

$$
\int_{\Omega} \int_{\square} b_{0} u_{1} d x d \mu=\int_{\Omega} \int_{\square} b f u_{1} d x d \mu
$$

и поэтому (8.9) следует из (8.7).

Наконец, выясним, когда точка $s \in\left\{\omega_{1}, \omega_{2}, \ldots\right\}$ будет собственным значением оператора $\mathscr{A}$. По определению множества $\left\{\omega_{1}, \omega_{2}, \ldots\right\}$ имеем $\mathscr{A}_{0} \varphi=s \varphi,\langle\varphi\rangle \neq 0$. Пусть $u=u_{1}(x)+v(x, y)-$ собственная функция оператора $\mathscr{A}$. Тогда из $(8.5)$ следует ортогональность

$$
\mathscr{A}_{0} v-s v=s u_{1} \perp \varphi \text { в } L^{2}\left(F_{0}, d \mu\right) .
$$

Отсюда $u_{1} \equiv 0$ и поэтому (см. второе уравнение $\left.(8.5)\right)\langle v\rangle=0$. Видим, что собственная функция - чисто блоховская. При этом $s$ должно быть кратным собственным значением оператора $\mathscr{A}_{0}$.

Итак, оператор $\mathscr{A}$ имеет чисто точечный спектр в том смысле, что его $c o б c m-$ венные функиии образуют полную в Н систему.

При этом спектр оператора $\mathscr{A}$ устроен следуюшим образом. Он состоит из спектра оператора $\mathscr{A}_{0}$, которьй разбивается на две непересекающиеся части $\left\{\alpha_{1}, \alpha_{2}, \ldots\right\},\left\{\omega_{1}, \omega_{2}, \ldots\right\}$, и бесконечной серии конечнократных собственных значений, сходящихся слева к точкам $\omega_{1}, \omega_{2}, \ldots$. Точки $\alpha_{1}, \alpha_{2}, \ldots$ - это такие собственные значения $\mathscr{A}_{0}$, у которых все соответствуюшие собственные функции 
имеют нулевое среднее. Они являются бесконечнократными собственными значениями самого оператора $\mathscr{A}$ и составляют тривиальную или число блоховскую часть $\operatorname{Sp} \mathscr{A}$.

Точка из $\left\{\omega_{1}, \omega_{2}, \ldots\right\}$ будет собственньм значением оператора $\mathscr{A}$, причем бесконечнократньм, только в том случае, когда она есть кратное собственное значение оператора $\mathscr{A}_{0}$.

Самое примечательное в геометрической структуре спектра оператора $\mathscr{A}$ - это наличие особого рода "лакун". На рис. 6 жирно отмечена бесконечная система интервалов, на которых функция $\beta(s)$ отрицательна. Эти интервалы могут содержать точки из $\left\{\alpha_{1}, \alpha_{2}, \ldots\right\}$, но все остальные точки $\mathrm{Sp} \mathscr{A}$ обязаны принадлежать дополнительным отрезкам, на которых $\beta \geqslant 0$. Это геометрическое расположение имеет место независимо от выбора области $\Omega$, поскольку функция $\beta(s)$ строится только по блоховскому оператору $\mathscr{A}_{0}$. В частности, если вместо ограниченной области $\Omega$ взять все пространство $\mathbb{R}^{N}$, то спектр оператора $\mathscr{A}_{1}$ заполнит положительную полуось, а нетривиальньй спектр $\mathscr{A}$-все отрезки, на которых $\beta \geqslant 0$, т.е. станет бесконечнозонньм.

8.2. Теперь можно вернуться к вопросу о сходимости спектра при усреднении.

Рассмотрим случай, когда $d \mu=d x$ - мера Лебега, а мягкая фаза $F_{0}$ дисперсна, например $F_{0}$ - периодическая система шаров, $\square \cap F_{0}=B$ - шар. Кроме того, для некоторого упрошения допустим, что мягкая фаза $\Omega_{0}^{\varepsilon}$ состоит только из шариков - компонент множества $F_{0}^{\varepsilon}$, целиком содержашихся в $\Omega$ (приграничные шарики отнесем к жесткой фазе $\Omega_{1}^{\varepsilon}$ ).

В этих условиях мы докажем следующую теорему о сходимости спектра.

Tеорема 8.1. Cnектр оператора $\mathscr{A}_{\varepsilon}$ сxодится no Хаусдорфу $к$ спектру оператора $\mathscr{A}$. Подробнее это означает:

1) для любого $s \in \mathrm{Sp} \mathscr{A}$ найдется последовательность $s_{\varepsilon} \in \operatorname{Sp} \mathscr{A}_{\varepsilon}$ такая, что $s_{\varepsilon} \rightarrow s$ nрu $\varepsilon \rightarrow 0$;

2) все конечные предельные точки последовательности $s_{\varepsilon} \in \operatorname{Sp} \mathscr{A}_{\varepsilon}$ принадлехсат $\mathrm{Sp} \mathscr{A}$.

Из сходимости по Хаусдорфу ясно, что спектр оператора $\mathscr{A}_{\varepsilon}$ содержит лакуны (близкие к лакунам в спектре оператора $\mathscr{A}$ ), причем число этих лакун растет до бесконечности при $\varepsilon \rightarrow 0$. Теорема 8.1 остается верной, если ограниченную область $\Omega$ заменить на $\mathbb{R}^{N}$ (доказательство более громоздкое и здесь не приводится). Следовательно, заключение о сушествовании лакун в спектре справедливо и для самосопряженного оператора $-\operatorname{div}\left(A_{\varepsilon} \nabla\right)$ в $L^{2}\left(\mathbb{R}^{N}\right)$.

Отметим, что свойство 1) сходимости по Хаус дорфу всегда обеспечивается сильной резольвентной сходимостью, в то время как более тонкое свойство 2) может отсутствовать. Дело здесь в следуюшем. Если $u_{\varepsilon}-$ нормированные в $L^{2}\left(\Omega, d \mu_{\varepsilon}\right)$ собственные функции, $\mathscr{A}_{\varepsilon} u_{\varepsilon}=s_{\varepsilon} u_{\varepsilon}$, причем $s_{\varepsilon} \rightarrow s, u_{\varepsilon} \stackrel{2}{\rightarrow} u$, то всегда $\mathscr{A} u=s u$. Однако предельная функция $u$ может оказаться нулевой, и тогда точка $s$ не обязана принадлежать $\mathrm{Sp} \mathscr{A}$.

Сейчас установим одно свойство компактности, из которого непосредственно следует свойство 2). 
ЛЕмма 8.2. Пусть

$$
\mathscr{A}_{\varepsilon} u_{\varepsilon}=s_{\varepsilon} u_{\varepsilon}, \quad \int_{\Omega} u_{\varepsilon}^{2} d x=1
$$

Eсли $s_{\varepsilon} \rightarrow s \notin \mathrm{Sp} \mathscr{A}_{0}$, то последовательность $u_{\varepsilon}$ компактна в смысле сильной двухмаситабной сходимости.

ДокАЗАТЕЛЬство. Имеем

$$
\begin{gathered}
u_{\varepsilon} \in H_{0}^{1}(\Omega), \quad-\operatorname{div}\left(A_{\varepsilon} \nabla u_{\varepsilon}\right)=s_{\varepsilon} u_{\varepsilon} \\
\int_{\Omega_{1}^{\varepsilon}} A_{1}\left(\varepsilon^{-1} x\right) \nabla u_{\varepsilon} \cdot \nabla u_{\varepsilon} d x+\varepsilon^{2} \int_{\Omega_{0}^{\varepsilon}} A_{0}\left(\varepsilon^{-1} x\right) \nabla u_{\varepsilon} \cdot \nabla u_{\varepsilon} d x=s_{\varepsilon} \int_{\Omega} u_{\varepsilon}^{2} d x=s_{\varepsilon} .
\end{gathered}
$$

Продолжим $\left.u_{\varepsilon}\right|_{\Omega_{1}^{\varepsilon}}$ на всю область $\Omega$ с условием

$$
\widetilde{u}_{\varepsilon} \in H_{0}^{1}(\Omega), \quad \int_{\Omega}\left|\nabla \widetilde{u}_{\varepsilon}\right|^{2} d x \leqslant C<\infty .
$$

Хорошо известно, что такое продолжение возможно [9], [10; гл. III]. Дополнительно всегда можно обеспечить соотношение

$$
-\operatorname{div} A_{0}\left(\varepsilon^{-1} x\right) \nabla \widetilde{u}_{\varepsilon}=0 \text { в } \Omega_{0}^{\varepsilon} .
$$

Тогда для разности $z_{\varepsilon}=u_{\varepsilon}-\widetilde{u}_{\varepsilon}$ получаем

$$
z_{\varepsilon} \in H_{0}^{1}\left(\Omega_{0}^{\varepsilon}\right), \quad \varepsilon^{2} \operatorname{div} A_{0}\left(\varepsilon^{-1} x\right) \nabla z_{\varepsilon}+s_{\varepsilon} z_{\varepsilon}=s_{\varepsilon} \widetilde{u}_{\varepsilon} \text { в } \Omega_{0}^{\varepsilon} .
$$

Из оценки $(8.10)$ видно, что последовательность $\widetilde{u}_{\varepsilon}$ компактна в $L^{2}(\Omega)$,

$$
\widetilde{u}_{\varepsilon} \rightarrow \widetilde{u} \text { в } L^{2}(\Omega),
$$

и требуется проверить, что последовательность $z_{\varepsilon}$ компактна в смысле сильной двухмасштабной сходимости.

Ниже мы имеем дело с последовательностями функций, заданных на $\Omega_{0}^{\varepsilon}$; продолжаем эти функции нулем на $\Omega$ и рассматриваем вопросы компактности. Например, правая часть (8.11) имеет вид $s_{\varepsilon} \chi_{0}^{\varepsilon} \widetilde{u}_{\varepsilon}$, где $\chi_{0}^{\varepsilon}$ - характеристическая функция множества $F_{0}^{\varepsilon}$. Из $(8.12)$ видно, что

$$
\chi_{0}^{\varepsilon}(x) \widetilde{u}_{\varepsilon}(x) \stackrel{2}{\rightarrow} \widetilde{u}(x) \chi_{0}(y) .
$$

Рассмотрим более общее уравнение

$$
v_{\varepsilon} \in H_{0}^{1}\left(\Omega_{0}^{\varepsilon}\right), \quad \varepsilon^{2} \operatorname{div} A_{0}\left(\varepsilon^{-1} x\right) \nabla v_{\varepsilon}+s_{\varepsilon} v_{\varepsilon}=f_{\varepsilon}, \quad f_{\varepsilon} \in L^{2}\left(\Omega_{0}^{\varepsilon}\right) .
$$

Нам потребуется следующее ключевое свойство:

$$
\begin{gathered}
\text { если } f_{\varepsilon} \stackrel{2}{\rightarrow} f \in H, \text { то } \\
v_{\varepsilon} \stackrel{2}{\rightarrow} v=v(x, y), \quad v \in L^{2}(\Omega, X), \quad-\mathscr{A}_{0} v+s v=f .
\end{gathered}
$$


(Напомним, что в нашем случае пространство $X$ отождествляется с $H_{0}^{1}(B), B=$ $\square \cap F_{0}$ - шар.)

Легко видеть, что из свойства (8.15) (которое будет доказано ниже) следует компактность $z_{\varepsilon}$. Действительно, согласно $(8.15),(8.13)$ имеем

$$
z_{\varepsilon} \stackrel{2}{\rightarrow} z, \quad-\mathscr{A}_{0} z+s z=s \widetilde{u} .
$$

Умножая уравнение (8.11) скалярно на $v_{\varepsilon}$, а уравнение (8.14) - на $z_{\varepsilon}$, получаем

$$
\int_{\Omega} z_{\varepsilon} f_{\varepsilon} d x=s_{\varepsilon} \int_{\Omega} v_{\varepsilon} \chi_{0}^{\varepsilon} \widetilde{u}_{\varepsilon} d x
$$

В силу (8.15) и сильной сходимости (8.13)

$$
\lim _{\varepsilon \rightarrow 0} \int_{\Omega} z_{\varepsilon}(x) f_{\varepsilon}(x) d x=s \int_{\Omega} \int_{F_{0}} v(x, y) \widetilde{u}(x) d x d y=\int_{\Omega} \int_{F_{0}} z(x, y) f(x, y) d x d y
$$

(на последнем шаге мы использовали предельные уравнения для $v$ и $z$ ). В частности, взяв $f_{\varepsilon}=z_{\varepsilon}$, получим

$$
\lim _{\varepsilon \rightarrow 0} \int_{\Omega} z_{\varepsilon}^{2}(x) d x=\int_{\Omega} \int_{F_{0}} z^{2}(x, y) d x d y
$$

и искомая двухмасштабная компактность $z_{\varepsilon}$ установлена.

Итак, нам остается доказать свойство сходимости (8.15).

Рассмотрим самосопряженный в $L^{2}\left(\Omega_{0}^{\varepsilon}\right)$ оператор $T_{\varepsilon}=-\varepsilon^{2} \operatorname{div}\left(\mathscr{A}_{0}\left(\varepsilon^{-1} x\right) \nabla\right)$, отвечающий задаче Дирихле в $\Omega_{0}^{\varepsilon}$. Так как $\Omega_{0}^{\varepsilon}$ состоит из отдельных шаров, то оператор $T_{\varepsilon}$ распадается и его спектр совпадает со спектром оператора $\mathscr{A}_{0}$ : $\operatorname{Sp} T_{\varepsilon}=\operatorname{Sp} \mathscr{A}_{0}$. В нашем случае $\mathscr{A}_{0}=-\operatorname{div}_{y} A_{0} \nabla_{y}$ - самосопряженный оператор, отвечающий задаче Дирихле в шаре $B=\square \cap F_{0}$. Поскольку $s_{\varepsilon}$ отделена от спектра $T_{\varepsilon}$, то

$$
\left\|\left(T_{\varepsilon}-s_{\varepsilon}\right)^{-1}\right\| \leqslant \frac{1}{\rho\left(s_{\varepsilon}, \operatorname{Sp} \mathscr{A}_{0}\right)} \leqslant C<\infty .
$$

Отсюда для решения $v_{\varepsilon}$ уравнения (8.14) получаем оценку

$$
\varepsilon^{2} \int_{\Omega}\left|\nabla v_{\varepsilon}\right|^{2} d x+\int_{\Omega} v_{\varepsilon}^{2} d x \leqslant C_{1}<\infty
$$

Без потери общности считаем, что имеет место двухмасштабная сходимость

$$
v_{\varepsilon} \rightarrow v, \quad \varepsilon \nabla v_{\varepsilon} \stackrel{2}{\rightarrow} \nabla_{y} v, \quad v \in L^{2}\left(\Omega, H_{0}^{1}(B)\right)
$$

В интегральном тождестве

возьмем

$$
-\int_{\Omega} \varepsilon^{2} A_{0}\left(\varepsilon^{-1} x\right) \nabla v_{\varepsilon} \cdot \nabla \psi_{\varepsilon} d x+s_{\varepsilon} \int_{\Omega} v_{\varepsilon} \psi_{\varepsilon} d x=\int_{\Omega} f_{\varepsilon} \psi_{\varepsilon} d x
$$

$$
\psi_{\varepsilon}(x)=\varphi(x) b(y), \quad y=\varepsilon^{-1} x, \quad \varphi \in C_{0}^{\infty}(\Omega), \quad b(y) \in C_{0}^{\infty}(B)
$$

(функция $b(y)$ рассматривается как периодическая). Предельный переход дает

$$
\begin{aligned}
-\int_{\Omega} \int_{\square} A_{0}(y) & \nabla_{y} v \cdot \nabla_{y} b(y) \varphi(x) d x d y \\
& +s \int_{\Omega} \int_{\square} v(x, y) \varphi(x) b(y) d x d y=\int_{\Omega} \int_{\square} f(x, y) \varphi(x) b(y) d x d y .
\end{aligned}
$$

Отсюда $\operatorname{div}_{y} A_{0} \nabla_{y} v+s u=f$, что и требовалось. 


\section{§9. Приложение: о тангенциальном градиенте}

9.1. Градиент функции из соболевского пространства $H_{\mathrm{per}}^{1}=H_{\mathrm{per}}^{1}(\square, d \mu)$ определен неоднозначно. Множество градиентов данной функции имеет структуру $\nabla u+g$, где $\nabla u$-некоторый градиент, а $g$-произвольный градиент нуля, $g \in \Gamma(0)$.

По определению $g \in L^{2}(\square, d \mu)^{N}=\left(L^{2}\right)^{N}$ есть градиент нуля, если выполнено соотношение (3.1). Из него следует, что $\Gamma=\Gamma(0)$ - подпространство векторного пространства $\left(L^{2}\right)^{N}$. Установим некоторые свойства этого подпространства.

i) Если $g \in \Gamma, a \in L^{\infty}$, то $a g \in \Gamma$.

Чтобы убедиться в этом, достаточно рассмотреть случай $a \in C_{\mathrm{per}}^{\infty}$. Тогда из (3.1) имеем

$$
a \varphi_{n} \stackrel{L^{2}}{\rightarrow} 0, \quad \nabla\left(a \varphi_{n}\right)=a \nabla \varphi_{n}+\nabla a \varphi_{n} \stackrel{\left(L^{2}\right)^{N}}{\longrightarrow} a g \Longrightarrow a g \in \Gamma
$$

ii) Пусть $Q$ - ортогональный проектор из $L^{2}(\square, d \mu)^{N}$ на Г. Тогда

$$
Q(a g)=a Q g \quad \forall g \in L^{2}(\square, d \mu)^{N}, \quad a \in L^{\infty}(\square, d \mu) .
$$

В самом деле, положив $\bar{g}=Q g$, имеем

$$
\bar{g} \in \Gamma, \quad g-\bar{g} \perp \Gamma .
$$

Но тогда по свойству і)

$$
a \bar{g} \in \Gamma, \quad\langle a(g-\bar{g}) \cdot h\rangle=0 \quad \forall h \in \Gamma,
$$

что и требуется.

iii) Множество $\Gamma \cap L^{\infty}(\square, d \mu)^{N}$ плотно в $\Gamma$.

Действительно, если $a_{n}$ - характеристическая функция множества $\{x \in \square$, $|g| \leqslant n\}$, то $a_{n} g \in \Gamma$ и $a_{n} g \rightarrow g$ при $n \rightarrow \infty$.

Лемма 9.1. Существует н-измеримое периодическое подпространство $D(x) \subset \mathbb{R}^{N}$ такое, ито

$$
\Gamma=\Gamma(0)=\left\{g \in L^{2}(\square, d \mu)^{N}: g(x) \in D(x)\right\} .
$$

ДоказАТЕЛЬСтво. Пусть $e^{1}, \ldots, e^{N}-$ естественный базис в $\mathbb{R}^{N}$. Положим

$$
\xi^{i}=Q e^{i}
$$

и определим $L(x)$ как линейную оболочку векторов $\xi^{1}(x), \ldots, \xi^{N}(x)$.

Обозначим через $B$ подпространство в $L^{2}(\square, d \mu)^{N}$, определенное правой частью (9.1). Требуется проверить, что $\Gamma=B$.

Пусть $g \in \Gamma \cap\left(L^{\infty}\right)^{N}$. Тогда по свойству ii)

$$
g=g_{1} e^{1}+\cdots+g_{N} e^{N}=Q g=g_{1} \xi^{1}+\cdots+g_{N} \xi^{N} \in B .
$$


В силу свойства іiі) и замкнутости $B$ получаем $\Gamma \subseteq B$. Проверим теперь, что $B \subseteq \Gamma$. Так как $\xi^{1}, \ldots, \xi^{N} \in \Gamma$, то по свойствам і), ii)

$$
a(x) \sum_{i=1}^{N} \lambda_{i} \xi^{i} \in \Gamma, \quad a \in L^{\infty}, \quad \lambda_{i} \in \mathbb{R}^{1}
$$

Покажем, что такие элементы плотны в $B$. Пусть

$$
b \in B, \quad\left\langle a(x) b(x) \cdot\left(\sum_{i=1}^{N} \lambda_{i} \xi^{i}\right)\right\rangle=0 .
$$

Поскольку $а$ произвольно, то для $\mu$-п.в. $x$

$$
b(x) \cdot \sum_{i=1}^{N} \lambda_{i} \xi^{i}(x)=0
$$

что дает $b(x)=0$. Лемма доказана.

Теперь мы можем уточнить предложение 3.1. Пусть $T(x)=(D(x))^{\perp}$.

ПРЕДЛОЖЕНИЕ 9.2. Всякая функиия из соболевского пространства $H_{\mathrm{per}}^{1}$ имеет единственный градиент, удовлетворяющий условию

$$
A(x) \nabla u(x) \in T(x)
$$

Действительно, достаточно для $\mu$-п.в. $x \in \square$ решить задачу

$$
c(x) \in D(x), \quad A(x)[\nabla u(x)+c(x)] \perp D(x)
$$

где $\nabla u(x)$ - какой-то градиент $u$. Тогда $\nabla u+c$ будет удовлетворять условию (9.2).

В частности, существует единственный тангенциальный градиент, т.е. удовлетворяющий условию $\nabla u \in T(x)$. Очевидно также, что если $\nabla u(x)$ - какой-то градиент $u$, а $P(x)$ - ортогональный проектор $\mathbb{R}^{N} \rightarrow T(x)$, то $P(x) \nabla u(x)$ будет тангенциальньм градиентом.

Резюмируем полученные результаты. Справедлива следующая теорема.

ТЕОРема 9.3. Существует $\mu$-измеримое периодическое подпространство $T(x)$ такое, что мнохсество градиентов любой функиии из $H_{\text {per }}^{1}$ имеет вид $\nabla u(x)+g(x)$, әде $\nabla u(x) \in T(x)$, a $g(x)$ - произвольный вектор из $L^{2}(\square, d \mu)^{N}$ такой, что $g(x) \in T^{\perp}(x)$.

Подпространство $T(x)$ называется тангенииальным в точке $x$, а $\nabla u$ - тангенциальным градиентом. 
9.2. В некоторых вопросах предпочтительно иметь дело исключительно с тангенциальным градиентом. Но вспомним, что интегральному тождеству (3.3) удовлетворяет функция $u(x)$ вместе со своим градиентом, подчиненным условию (3.4), а не тангенциальњым. В связи с этим возникает задача так изменить исходную матрицу $A(x)$, чтобы новому уравнению с матрицей $\widehat{A}(x)$ удовлетворяла та же функция $u(x)$ вместе со своим тангенциальным градиентом. Эта процедура носит название релаксации; смысл названия будет ясен из дальнейшего.

Благодаря теореме 9.3 задача нахождения матрицы $\widehat{A}=\widehat{A}(x)$ носит поточечныцй характер. Положим

$$
\widehat{A}(x) \xi \cdot \xi=\min _{\eta \in D(x)} A(x)(\xi+\eta) \cdot(\xi+\eta) .
$$

Симметрическая матрица $\widehat{A}$ равна нулю на $D$ и имеет $T$ в качестве области значений, в частности $\widehat{A} \xi=\widehat{A} P \xi$. Кроме того, $A \xi=\widehat{A} \xi$, если $A \xi \in T$. Действительно, в этом случае

$$
\begin{gathered}
A(\xi+\eta) \cdot(\xi+\eta)=A \xi \cdot \xi+A \eta \cdot \eta, \\
\widehat{A} \xi \cdot \xi=A \xi \cdot \xi \Longrightarrow A \xi=\widehat{A} \xi(\text { поскольку } A \geqslant \widehat{A}) .
\end{gathered}
$$

Возвратимся теперь к тождеству (3.3) и вспомним, что $A \nabla u \in T$. В результате

$$
A \nabla u \cdot \nabla \varphi=\widehat{A} \nabla u \cdot \nabla \varphi=\widehat{A} P \nabla u \cdot \nabla \varphi
$$

и поскольку $P \nabla u$ - тангенциальный градиент, задача релаксации решена.

Вектор-функцию $b(x)$ будем называть тангенциальной, если $b(x) \in T(x)$. Из определения матрицы $\widehat{A}$ видно, что вектор $\widehat{A}(x) \xi$ тангенциален. Все собственные векторы матрицы $\widehat{A}$ лежат в тангенциальном подпространстве.

ПРИМЕР. Пусть $N=2$,

$$
A=\left(\begin{array}{ll}
a_{11} & a_{12} \\
a_{12} & a_{22}
\end{array}\right)>0
$$

$T-$ ось $x_{1}$. Тогда

$$
\widehat{A}=\left(\begin{array}{cc}
a_{11}-\frac{a_{12}^{2}}{a_{22}} & 0 \\
0 & 0
\end{array}\right) .
$$

Термин “релаксация" связан со следующим обстоятельством. Функционал

$$
\Phi(u)=\langle A(x) \nabla u \cdot \nabla u\rangle \text { на } C_{\text {per }}^{\infty}
$$

не является полунепрерывным снизу относительно $L^{2}$-сходимости. Его релаксация

$$
\widehat{\Phi}(u)=\inf _{u_{\varepsilon} \rightarrow u} \Phi\left(u_{\varepsilon}\right)=\min _{\nabla u \in \Gamma(u)}\langle A(x) \nabla u \cdot \nabla u\rangle=\langle\widehat{A} \nabla u \cdot \nabla u\rangle .
$$

Последний интеграл справа уже не зависит от выбора градиента, и можно взять в нем тангенциальный градиент.

Лемма 9.1 доказана совместно с профессором Dal Maso, которому автор выражает благодарность за обсуждение вопросов, касающихся градиента. По поводу проблемы релаксации см. также [25]. 
9.3. Сделаем несколько замечаний относительно потенциальных и соленоидальных векторов. Очевидно, что любой градиент нуля есть потенциальный вектор. Напомним (см. (3.7)), что $v$ потенциален, $v \in V_{\text {pot }}$, если

$$
\exists \varphi_{n} \in C_{\text {per }}^{\infty}: \nabla \varphi_{n} \rightarrow v \text { в }\left(L^{2}\right)^{N} .
$$

Разлагая $\nabla \varphi_{n}$ в ортогональную сумму $\nabla \varphi_{n}=P \nabla \varphi_{n}+a_{n}, a_{n} \in \Gamma$, приходим к очевидному представлению

$$
V_{\text {pot }}=\widehat{V}_{\text {pot }} \oplus \Gamma
$$

где $\widehat{V}_{\text {pot }}$ есть замькание в $\left(L^{2}\right)^{N}$ множества $\left\{P \nabla \varphi, \varphi \in C_{\text {per }}^{\infty}\right\}$. Элементы из $\widehat{V}_{\text {pot }}-$ это тангенциальные потенциальные векторы.

Любопытно, что соленоидальные векторы автоматически тангенциальны. Действительно, пусть вектор $b$ соленоидален, т.е. выполнено условие ортогональности (3.8). Выбрав в (3.8) $\varphi=\varphi_{n}-$ последовательность из $(3.1)$, получим $b \perp \Gamma(0)$. Отсюда следует наше утверждение.

Отмеченное свойство позволяет описать соленоидальные векторы в случае периодического графа на плоскости. Соленоидальный вектор на каждом ребре направлен вдоль ребра и постоянен на этом ребре (последнее легко следует из (3.8)). Рассмотрим вершину графа. Пусть в ней сходится $s$ ребер. Возьмем единичные векторы $b^{1}, b^{2}, \ldots, b^{s}$, идущие вдоль ребер к вершине. Тогда на ребрах вектор $b$ равен $\lambda_{1} b^{1}, \lambda_{2} b^{2}, \ldots, \lambda_{s} b^{s}$, причем должно быть выполнено соотношение

$$
\lambda_{1}+\lambda_{2}+\cdots+\lambda_{s}=0
$$

легко вытекающее из (3.8). Такие соотношения нужно составить для каждой вершины, приняв во внимание условие периодичности. Дело сведется к решению некоторой однородной системы линейных уравнений.

\section{Список литературы}

1. De Giorgi E., Spagnolo S. Sulla convergenza degli integrali dell'energia per operatori ellittici del secondo ordine // Boll. Unione Mat. Ital. 1973. № 8. P. 391-411.

2. Бахвалов Н. С. Осреднение дифференциальных уравнений с частньми производными с быстро осциллирующими коэффицциентами // Докл. АН СССР. 1975. Т. 221. №3. C. $516-519$.

3. Бахвалов Н. С., Панасенко Г. П. Осреднение процессов в периодических средах. М.: Наука, 1984.

4. Nguetseng G. A general convergence result for a functional related to the theory of homogenization // SIAM J. Math. Anal. 1989. V. 20. P. 608-623.

5. Allaire G. Homogenization and two-scale convergence // SIAMJ. Math. Anal. 1992. V. 23. P. $1482-1518$

6. Жиков В. В. О весовых соболевских пространствах // Матем. сб. 1998. Т. 189. №8. C. $27-58$.

7. Жиков В. В. Связность и усреднение. Примеры фрактальной проводимости // Матем. сб. 1996. Т. 187. № 8 . С. 3-40.

8. Жиков В. В. К технике усреднения вариационных задач // Функц. анализ и его прилож. 1999. T. 33. № 1. C. 14-29.

9. Жиков В.В., Козлов С. М., Олейник О. А. Усреднение дифференциальных операторов. М.: Наука, 1993.

10. Zhikov V.V., Kozlov S. M., Olejnik O.A. Homogenization of differential operators and integral functionals. Berlin: Springer-Verlag, 1994. 
11. Олейник О.А., Иосифьян Г. А., Шамаев А. С. Математические задачи теории сильно неоднородных упругих сред. М.: Изд-во МГУ, 1990.

12. Arbogast T., Douglas J., Hornung U. Derivation of the double porosity model of single phaseflow via homogenization theory // SIAM J. Math. Anal. 1990. V. 21. № 4. P. 823-836.

13. Hornung U., Jäger $W$. Diffusion, convection, adsorption, and reaction of chemicals in porous media // J. Differential Equations. 1991. V. 92. № 2. P. 199-225.

14. Bourgeat A., Mikelič A., Pyatnitski A. Modèle de double porosité aléatoire // C. R. Acad. Sci. Paris Sér. I Math. 1998. V. 327. P. 99-104.

15. Hornung U. (ed.) Homogenization and porous media. New York: Springer-Verlag, 1997.

16. Сандраков Г. В. Осреднение нестационарных уравнений с контрастньми коэффициентами // Докл. РАН. 1997. Т. 335. № 5. С. 605-608.

17. Сандраков Г. В. Осреднение нестационарных задач теории сильно неоднородных упругих сред // Докл. РАН. 1998. Т. 358. № 3. С. 308-311.

18. Экланд Н., Темам Р. Вьпукльй анализ и вариационные проблемы. М.: Мир, 1979.

19. Allaire G., Damlamian A., Hornung U. Two-scale convergence on periodic surfaces and applications // Mathematical modelling of flow through porous media / ed. A. Bourgeat, C. Carasso, S. Luckhaus, A. Mikelić. Singapore: World Scientific, 1995. P. 15-25.

20. Neuss-Radu M. Some extensions of two-scale convergence // C. R. Acad. Sci. Paris Sér. I Math. 1996. V. 322. P. 899-904.

21. Като Т. Теория возмущений линейных операторов. М.: Мир, 1972.

22. Khruslov E. Ya. Homogenized models of strongly inhomogeneous media // Proceedings of the International congress of mathematicians, Zurich, Switzerland, 1994. Basel: Birkhäuser, 1995.

23. Хруслов E. Я. Усреднение модели сильно неоднородных сред с памятью // УМН. 1990. T. 45. №1. C. 197-199.

24. Khruslov E. Homogenized models of composite media // Composite media and homogenization theory / ed. G. Dal Maso, G.F. Dell'Antonio. Basel: Birkhäuser, 1991. P. 159-182.

25. Bouchitté G., Buttazzo G., Seppecher P. Energies with respect to a measure and applications to low dimensional structures // Calc. Var. Partial Differential Equations. 1997. V. 5. № 1. P. 37-54.

Владимирский государственнњй

Поступила в редакцию педагогический университет 19.04.1999 и 17.02 .2000

E-mail: zhikov@vgpu.vladimir.ru 